\title{
QUANTIZATION OPERATORS ON QUADRICS
}

\author{
Wolfram BAUER and Kenro FURUTANI
}

(Received 12 March 2007)

\begin{abstract}
We explain the construction of a Hilbert space on quadrics arising by the method of pairing polarizations. Then we introduce a family of measures and operators from function spaces on these quadrics to $L_{2}\left(S^{n}\right)$ which are defined by fiber integration. We compare the quantization operators and characterize them in the framework of pseudo-differential operator theory. An asymptotic property of the reproducing kernel of the Hilbert spaces consisting of holomorphic functions defined on quadrics is proved. This is a generalization of the SegalBargmann space and its reproducing kernel. Next we treat the case of the complex projective space and we explain that the space corresponding to the quadric is a matrix space consisting of rank-one complex matrices whose square is zero. Most of the theorems can be stated in the same way parallel to the sphere case.
\end{abstract}

\section{Contents}

1 Introduction $\quad 221$

2 Hilbert space on quadrics $\quad 223$

3 Comparison of quantization operators $\quad 227$

4 Reproducing kernel $\quad \mathbf{2 3 2}$

5 Geodesic flow and quantization of a free particle 237

6 Complex projective space $\quad 238$

7 Proofs of Theorems 6.8, 6.9 and reproducing kernel $\quad 244$

8 Complexified Hopf fibration and quantization operators $\quad 251$

$\begin{array}{ll}\text { References } & 257\end{array}$

\section{Introduction}

For any manifold we have two naturally defined spaces, its cotangent (tangent) bundle and the space of functions on it. When we consider a manifold as a set of a certain kind of physical states (the configuration space, which will have a manifold structure), the functions on it are said to be quantum states and the functions on the cotangent bundle are thought of as the observables in the sense of classical mechanics. In the most naive sense, we understand that a correspondence between quantities on the cotangent bundle and some quantity of the quantum states or quantum states themselves is a kind of quantization.

2000 Mathematics Subject Classification: Primary 53D50, 53D25, 32Q15.

Keywords and Phrases: geometric quantization; pairing of the polarizations; Kähler structure; sphere; complex projective space; geodesic flow; Hopf fibration; reproducing kernel; Segal-Bargmann space. 
Now, let $\mathcal{F}^{0}(M)$ and $\mathcal{P}^{0}(M)$ be the group of invertible Fourier integral operators and the group of invertible zeroth-order (classical-type) pseudo-differential operators on a closed manifold $M$ respectively. Then we have a well-known exact sequence

$$
\{0\} \longrightarrow \mathcal{P}^{0}(M) \longrightarrow \mathcal{F}^{0}(M) \stackrel{\sigma}{\rightarrow} \operatorname{Diff}_{\text {sym }}^{1}\left(T_{0}^{*}(M)\right) \longrightarrow\{0\},
$$

where $\operatorname{Diff}_{\text {sym }}^{1}\left(T_{0}^{*}(M)\right.$ ) denotes the group of homogeneous (of degree 1) symplectic isomorphisms of the punctured cotangent bundle $T_{0}^{*}(M)$. The map

$$
\mathcal{F}^{0}(M) \stackrel{\sigma}{\rightarrow} \operatorname{Diff}_{\text {sym }}^{1}\left(T_{0}^{*}(M)\right)
$$

is the assignment of a Fourier integral operator to its symplectic isomorphism whose graph is the canonical relation of the Fourier integral operator (cf. [16-18]). An inverse group homomorphism $\operatorname{Diff}_{\text {sym }}^{1}\left(T_{0}^{*}(M)\right) \longrightarrow \mathcal{F}^{0}(M)$ can be understood as a kind of quantization. However, we will not have such a map defined on all of the group $\operatorname{Diff}_{\text {sym }}^{1}\left(T_{0}^{*}(M)\right)$, so we must restrict ourselves to a subgroup of $\operatorname{Diff}_{\text {sym }}^{1}\left(T_{0}^{*}(M)\right)$ to be able to define an inverse map (see [8] for the case of compact subgroups of $\operatorname{Diff}_{\text {sym }}^{1}\left(T_{0}^{*}(M)\right)$ ). Of course there is a typical subgroup namely the group of diffeomorphisms of the manifold $M$, which can be seen as a subgroup of the Fourier integral operators $\mathcal{F}^{0}(M)$ and also can be naturally lifted to a subgroup of $\operatorname{Diff}_{\text {sym }}^{1}\left(T_{0}^{*}(M)\right)$. Note that a homogeneous symplectic isomorphism which is defined on all of the cotangent bundle arises from a diffeomorphism of the underlying manifold $M$ (cf. [29]). However, this example will not be so interesting, since it does not depend on any additional structure of the manifold. There would be one way to construct such a map, if we have a Hilbert space $\mathcal{H}$ of 'nice' classical observables and an isomorphism $T: \mathcal{H} \longrightarrow L_{2}(M)$ such that for a certain class of symplectic isomorphisms $C \in \operatorname{Diff}_{\omega}^{1}\left(T_{0}^{*}(M)\right)$, which must preserve $\mathcal{H}$, the operator $T \circ C \circ T^{-1}$ will be in the group $\mathcal{F}^{0}(M)$ and $\sigma\left(T \circ C \circ T^{-1}\right)=C$. If the cotangent bundle or the punctured cotangent bundle has a complex structure, then the nice classical observables will consist of holomorphic functions. This is the case we discuss in this paper. We explain the method of pairing polarizations in the case of the sphere, following [23] as a typical and simple example, and we gather up the cases for which the punctured cotangent bundle has a Kähler structure. In general, it will not be trivial to construct such a Kähler structure on the (punctured) cotangent bundle whose Kähler form coincides with the natural symplectic form (cf. [2022, 24-27]). Here we treat the cases of spheres and complex projective spaces for which such a Kähler structure was constructed in [24], [14] and [12]. Based on this Kähler structure we can consider various Hilbert spaces consisting of holomorphic functions. In [23], [15] and [11] an operator was defined which gives an isomorphism between such a Hilbert space of holomorphic functions and the $L_{2}$-space on the base manifold (the configuration space) by the method of pairing polarizations (cf. [2]). A priori, inner products and fiber integration should be determined by this method. In the present paper, we define various Hilbert spaces consisting of holomorphic functions and quantization operators by inventing new parameters $(h, N)$ to these inner products. We specify the parameters leading to isomorphisms among the quantization operators and we compare their differences coming from the variation of $(h, N)$. Then we make it clear that most of the phenomena occurring are similar in the cases of the sphere, complex projective space and quaternion projective space. In this paper we do not treat the case of the quaternion projective space and the Cayley projective plane. However, we 
have already constructed an explicit Kähler structure on the punctured cotangent bundle of the Cayley projective plane by embedding it into an $8 \times 8$-complex matrix space (cf. [12]). As for the case of quaternion projective spaces we have not only obtained a Kähler structure on the punctured cotangent bundle, but have also constructed the pairing of polarizations explicitly in [11]. Moreover, in [13] we have proved a theorem [13, Theorem 4.2] corresponding to Theorem 8.5 in this paper. As for these two cases several results corresponding to those in the present paper (cf. Theorems 3.1, 3.5, 4.2, 7.5 and so on) will be written separately.

In Section 2 we explain the construction of a Hilbert space on quadrics defined by the method of pairing polarizations. We follow [23] and based on the data arising from this example we introduce a family of measures and operators from function spaces on these quadrics to $L_{2}\left(S^{n}\right)$, which are defined by fiber integration. In Section 3 we compare the quantization operators and characterize them in the framework of a class of pseudodifferential operators. In Section 4 we prove an asymptotic property of the reproducing kernel of the Hilbert spaces consisting of holomorphic functions defined on quadrics. This is a generalization of the Segal-Bargmann space and its reproducing kernel (cf. [2, 4]). The case of the complex projective space is treated in Sections 5 and 6 and the space corresponding to the quadrics above consists of rank-one complex matrices whose square is zero. This is also a non-singular affine variety which is isomorphic to the punctured cotangent bundle of the complex projective space. Most of the theorems can be stated in a way parallel to the sphere case.

\section{Hilbert space on quadrics}

Let $S^{n}=\left\{\left(x_{0}, \ldots, x_{n}\right) \in \mathbb{R}^{n+1} \mid \sum x_{k}^{2}=1\right\}$ be the $n$-dimensional sphere with the standard Riemann metric induced from the Euclidean metric on $\mathbb{R}^{n+1}$. We identify the tangent bundle $T\left(S^{n}\right)$ and the cotangent bundle $T^{*}\left(S^{n}\right)$ by this metric and realize them as a subspace in $\mathbb{R}^{n+1} \times \mathbb{R}^{n+1}$ :

$$
\begin{aligned}
T^{*}\left(S^{n}\right) & \cong T\left(S^{n}\right) \\
& =\left\{(x, y) \in \mathbb{R}^{n+1} \times \mathbb{R}^{n+1} \mid \sqrt{\sum x_{k}^{2}}=\|x\|=1,(x, y)=\sum x_{k} y_{k}=0\right\} .
\end{aligned}
$$

The symplectic form $\omega_{S}$ and the canonical one form $\theta_{S}$ on $T^{*}\left(S^{n}\right)$ are then the restrictions of the symplectic form $\sum_{k=0}^{n} d y_{k} \wedge d x_{k}$ and the canonical one form $\sum_{k=0}^{n} y_{k} d x_{k}$ on $T^{*}\left(\mathbb{R}^{n+1}\right) \cong \mathbb{R}^{n+1} \times \mathbb{R}^{n+1}$, respectively.

Let $T_{0}^{*}\left(S^{n}\right) \cong \mathbf{E}_{S}=\left\{(x, y) \in T\left(S^{n}\right) \mid\|x\|=1, y \neq 0\right\}$ be the punctured cotangent bundle. Then we define the map

$$
\tau_{S}: \mathbf{E}_{S} \longrightarrow \mathbb{C}^{n+1} \backslash\{0\}, \quad(x, y) \mapsto z=\|y\| x+\sqrt{-1} y
$$

which gives a diffeomorphism onto

$$
\tau_{S}\left(\mathbf{E}_{S}\right)=\mathbf{X}_{S}=\left\{z \in \mathbb{C}^{n+1} \mid \sum_{k=0}^{n} z_{k}^{2}=0, z \neq 0\right\} .
$$

Through this map it can be checked that the canonical one form $\theta_{S}$ and the symplectic form $\omega_{S}$ are expressed as

$$
\theta_{S}=\sqrt{\frac{-1}{2}}(\partial\|z\|-\bar{\partial}\|z\|)
$$


and

$$
\omega_{S}=d \theta_{S}=\sqrt{-2} d \partial\|z\|=\sqrt{-2} \bar{\partial} \partial\|z\| .
$$

First we look over the construction of a Hilbert space consisting of holomorphic functions on quadrics $\mathbf{X}_{S}$ given in [23] and an operator (called the quantization operator of the geodesic flow) from this Hilbert space to $L_{2}\left(S^{n}\right)$ (see $\left.[\mathbf{1 9}, \mathbf{2 0}, 24]\right)$.

There are two global trivializations of the line bundle $(L, \nabla)$ defined by the symplectic form $\omega_{S}$ and the global sections $\mathbf{s}_{S}$ and $\mathbf{t}_{S}$ such that the connection $\nabla$ is given by the formulas

$$
\nabla_{\xi}\left(\mathbf{s}_{S}\right)=2 \pi \sqrt{-1}\left\langle\theta_{S}, \xi\right\rangle \mathbf{s}_{S}
$$

and

$$
\nabla_{\xi}\left(\mathbf{t}_{S}\right)=2 \pi \sqrt{-1}\langle\sqrt{-2} \partial\|z\|, \xi\rangle \mathbf{t}_{S}=-2 \sqrt{2} \pi\langle\partial\|z\|, \xi\rangle \mathbf{t}_{\mathbf{S}} .
$$

If we put $\mathbf{t}_{S}=\varphi \mathbf{s}_{S}$, then the function $\varphi$ satisfies the equation

$$
d \log \varphi=2 \pi \sqrt{-1}\left(\sqrt{-2} \partial\|z\|-\theta_{S}\right)=\sqrt{-2} \pi d\|z\|,
$$

and the solution is given by (mod constants)

$$
\varphi(z)=e^{-\sqrt{2} \pi\|z\|} .
$$

We introduce an inner product on the trivial line bundle $L$ with $\left\langle\mathbf{s}_{S}, \mathbf{s}_{S}\right\rangle \equiv 1$. Then the norm of the section $\mathbf{t}_{S}$ is $\sqrt{\left\langle\mathbf{t}_{S}, \mathbf{t}_{S}\right\rangle}=e^{-\sqrt{2} \pi\|z\|}$.

Let $d \mathrm{vol}_{S}$ be the Riemann volume form on the sphere, then it is expressed as

$$
d \operatorname{vol}_{S}=\sum_{k=0}^{n}(-1)^{k} x_{k} d x_{0} \wedge \cdots \wedge d \hat{x}_{k} \wedge \cdots \wedge d x_{n} .
$$

Moreover, the pull-back $\pi_{S}^{*}\left(d \operatorname{vol}_{S}\right)$ of the volume element $d \mathrm{vol}_{S}$ by the projection map $\pi_{S}: T_{0}^{*}\left(S^{n}\right) \rightarrow S^{n}$ gives the trivialization of the canonical line bundle $K^{F}$ of the real polarization $F\left(=\operatorname{Ker}\left(d \pi_{S}\right) \subset T\left(T_{0}^{*} S^{n}\right)\right)$.

The Kähler polarization $G$ (anti-holomorphic tangent vectors) on $\mathbf{X}_{S}$ defined by the embedding $\tau_{S}$ satisfies

$$
\langle\partial\|z\|, G\rangle=0 .
$$

We have a nowhere vanishing holomorphic global section

$$
\sigma_{S}=\frac{2}{\|z\|^{2}} \sum_{k=0}^{n}(-1)^{k} \bar{z}_{k} d z_{0} \wedge \cdots \wedge d \hat{z}_{k} \wedge \cdots \wedge d z_{n}
$$

of the canonical line bundle $K^{G}$ (the canonical line bundle $K^{G}$ is the highest exterior product of the annihilator $\left.G^{\circ}=\left\{\xi \in T^{*}\left(\mathbf{X}_{S}\right) \otimes \mathbb{C} \mid \xi(G)=0\right\}\right)$.

Let

$$
\Omega_{S}=\frac{(-1)^{n(n-1) / 2}}{n !} \omega_{S}
$$

be the Liouville volume form on $T_{0}^{*}\left(S^{n}\right)$, then we have the pairings as follows:

$$
\begin{aligned}
\pi_{S}^{*}\left(d \operatorname{vol}_{S}\right) & \wedge \sigma_{S}=\sqrt{2} \sqrt{-1}^{n}\|z\|^{-1} \Omega_{S}, \\
\sigma_{S} & \wedge \overline{\sigma_{S}}=2^{n / 2+3}\|z\|^{n-2} \Omega_{S} .
\end{aligned}
$$


By taking the square roots of these data, an inner product of the polynomials on $\mathbb{C}^{n+1}$ restricted to the quadrics is defined by the integral

$$
\left\langle p_{1}(z), p_{2}(z)\right\rangle=\int_{\mathbf{X}_{S}} p_{1}(z) \overline{p_{2}(z)} e^{-2 \sqrt{2} \pi\|z\|}\|z\|^{n / 2-1} \Omega_{S} .
$$

Furthermore, the pairing of sections $f \cdot \mathbf{s}_{S} \otimes \sqrt{\pi_{S}^{*}\left(d \operatorname{vol}_{S}\right)} \in \Gamma_{F}\left(L \otimes \sqrt{K^{F}}\right)$ and $p \cdot \mathbf{t}_{S} \otimes \sqrt{\sigma_{S}} \in \Gamma_{G}\left(L \otimes \sqrt{K^{G}}\right)$ is given by the integral

$$
\int_{\mathbf{X}_{S}} f\left(\pi_{S} \circ \tau_{S}^{-1}(z)\right) p(z) e^{-\sqrt{2} \pi\|z\|}\|z\|^{-1 / 2} \Omega_{S} .
$$

Here we mean a section $\psi \in \Gamma_{G}\left(L \otimes \sqrt{K^{G}}\right), \psi=f \cdot \mathbf{t}_{S} \otimes \sqrt{\sigma_{S}}$, if the coefficient function $f$ is holomorphic on $\mathbf{X}_{S}$ (see [23] and [30] for the definition in terms of the connection along the polarization).

The transformation $\mathbf{T}_{S}$ from the space of parallel sections $\Gamma_{G}\left(L \otimes \sqrt{K^{G}}\right)$ to $C^{\infty}\left(S^{n}\right)$ is given by fiber integration:

$$
\mathbf{T}_{S}(p)(x) d \operatorname{vol}_{S}(x)=\left(\pi_{S}\right)_{*}\left(p(z) e^{-\sqrt{2} \pi\|z\|}\|z\|^{-1 / 2} \Omega_{S}\right), \quad x=\pi_{S}(z) .
$$

More precisely, let $f \in C^{\infty}\left(S^{n}\right)$, then the integral

$$
C^{\infty}\left(S^{n}\right) \ni f \mapsto \int_{\mathbf{X}_{S}}\left(\pi_{S} \circ \tau_{S}^{-1}\right)^{*}(f)(z) p(z) e^{-\sqrt{2} \pi\|z\|}\|z\|^{-1 / 2} \Omega_{S}
$$

defines a distribution on $S^{n}$. Since the map $\pi_{S}$ is a submersion, this distribution is given by a smooth function which we denote by

$$
\mathbf{T}_{S}(p)(x)=*\left(\left(\pi_{S}\right)_{*}\left(p(z) e^{-\sqrt{2} \pi\|z\|}\|z\|^{-1 / 2} \Omega_{S}\right)\right),
$$

where $*$ is the Hodge $*$-operator.

With these data we make completion of the space of polynomials on $\mathbf{X}_{S}$ (and denote it by $\mathcal{H}^{2}\left(\mathbf{X}_{S}\right)$ ) with respect to the above inner product.

THEOREM 2.1. (Rawnsley [23]) We have that

$$
\mathbf{T}_{S}: \mathcal{H}^{2}\left(\mathbf{X}_{S}\right) \rightarrow L_{2}\left(S^{n}\right)
$$

is an isomorphism.

From now on we always identify the space of parallel sections $\Gamma_{G}\left(L \otimes \sqrt{K^{G}}\right)$ (with respect to the connection of the line bundle $L$ given in the formula (2.3) and that along the Kähler polarization $G$ ) with the space of holomorphic functions $\phi$ on quadrics through the correspondence

$$
\phi \longleftrightarrow \phi \cdot \mathbf{t}_{S} \otimes \sqrt{\sigma_{S}}
$$

and that in $\Gamma_{F}\left(L \otimes \sqrt{\left(\pi_{S}\right)^{*}\left(d \operatorname{vol}_{S}\right)}\right)$ with a smooth functions on the sphere. We introduce a family of new inner products on the space of polynomials on $\mathbb{C}^{n+1}$ restricted to the quadrics $\mathbf{X}_{S}$ and depending on two parameters $(h, N), h>0$ and $N>-n$ as follows:

$$
\left\langle p_{1}, p_{2}\right\rangle_{(h, N)}=\int_{\mathbf{X}_{S}} p_{1}(z) \overline{p_{2}(z)} e^{-h\|z\|}\|z\|^{N} \Omega_{S} .
$$


When we decompose the quadrics as

$$
\mathbf{X}_{S} \cong \mathbb{R}_{+} \times \Sigma(S), \quad \Sigma(S)=\mathbf{X}_{S} \cap\left\{z \in \mathbb{C}^{n+1} \mid\|z\|=1\right\}
$$

the Liouville volume form $\Omega_{S}$ is expressed as

$$
\Omega_{S}=t^{n-1} d t \wedge d \mathrm{v}_{\Sigma(S)}(\sigma), \quad(t, \sigma) \in \mathbb{R}_{+} \times \Sigma(S)
$$

with a nowhere vanishing $(2 n-1)$-form $d v_{\Sigma(S)}$ on $\Sigma(S)$ (volume form on $\Sigma(S)$ ) which is invariant under the action (lifted to $\mathbf{X}_{S}$ ) of the orthogonal group $O(n)$. By the condition $N>-n$, all polynomials (restricted to $\mathbf{X}_{S}$ ) are square-integrable with respect to the measure

$$
d m_{(h, N)}(z)=e^{-h\|z\|}\|z\|^{N} \Omega_{S}(z) .
$$

Definition 2.2. We write $\mathcal{H}^{2}\left(\mathbf{X}_{S}, d m_{(h, N)}(z)\right)$ for the Hilbert space completion of the polynomials on $\mathbf{X}_{\mathbb{C}}$ and with respect to the inner product (2.6).

In terms of fiber integration and corresponding to the (2.7) we introduce an operator $T_{(h, N)}^{S}$ from functions on $\mathbf{X}_{S}$ with a suitable integrability condition to functions on $S^{n}$.

Definition 2.3. We have

$$
T_{(h, N)}^{S}(\varphi)(x) d \operatorname{vol}_{S}(x)=\left(\pi_{S} \circ \tau_{S}^{-1}\right)_{*}\left(\varphi(z) d m_{(h, N)}(z)\right), \quad \pi_{S} \circ \tau_{S}^{-1}(z)=x .
$$

One of our main purposes in the present paper is to give a relation among the parameter $(h, N)$ and $\left(h^{\prime}, N^{\prime}\right)$ which leads to isomorphisms $T_{\left(h^{\prime}, N^{\prime}\right)}^{S}$ between both Hilbert spaces $\mathcal{H}^{2}\left(\mathbf{X}_{S}, d m_{(h, N)}(z)\right)$ and $L_{2}\left(S^{n}\right)$.

There is a version of Liouville's theorem for holomorphic functions on a quadric.

PROPOSITION 2.4. Let $n \geq 2$, then there are no bounded non-constant holomorphic functions on the quadrics $\mathbf{X}_{S}$.

For the proof see [22]. In this note we always assume that $n \geq 2$.

All functions in $\mathcal{H}^{2}\left(\mathbf{X}_{S}, d m_{(h, N)}(z)\right)$ can be expanded into an infinite sum as follows.

Proposition 2.5. The function $\varphi \in \mathcal{H}^{2}\left(\mathbf{X}_{S}, d m_{(h, N)}(z)\right)$ has an expansion of the form $\varphi(z)=\sum_{k=0}^{\infty} \varphi_{k}(z)$ with kth-order polynomials $\varphi_{k}$.

The norm of $\varphi(z)=\sum_{k=0}^{\infty} \varphi_{k}(z) \in \mathcal{H}^{2}\left(\mathbf{X}_{S}, d m_{(h, N)}(z)\right)$ can be expressed as

$$
\begin{aligned}
\|\varphi\|_{(h, N)}^{2} & =\int_{\mathbf{X}_{S}}|\varphi(z)|^{2} d m_{(h, N)}(z) \\
& =\sum_{k=0}^{\infty} \int_{\Sigma(S)}\left|\varphi_{k}(\sigma)\right|^{2} d \mathrm{v}_{\Sigma(S)}(\sigma) \times \int_{0}^{\infty}\|z\|^{2 k+N+n-1} e^{-h\|z\|} d\|z\| \\
& =\sum_{k=0}^{\infty} \frac{\Gamma(2 k+N+n)}{h^{2 k+N+n}} \int_{\Sigma(S)}\left|\varphi_{k}(\sigma)\right|^{2} d \mathrm{v}_{\Sigma(S)}(\sigma)
\end{aligned}
$$

and we have the following. 
Proposition 2.6. Let $-n<\tilde{N} \leq N$, then

$$
\mathcal{H}^{2}\left(\mathbf{X}_{S}, d m_{(h, N)}(z)\right) \hookrightarrow \mathcal{H}^{2}\left(\mathbf{X}_{S}, d m_{(h, \tilde{N})}(z)\right)
$$

and the inclusion is continuous: let $\varphi \in \mathcal{H}^{2}\left(\mathbf{X}_{S}, d m_{(h, N)}(z)\right)$, then

$$
\begin{aligned}
\|\varphi\|_{(h, \tilde{N})}^{2} & =\sum_{k=0}^{\infty} \frac{\Gamma(2 k+\tilde{N}+n)}{h^{2 k+\tilde{N}+n}} \int_{\Sigma(S)}\left|\varphi_{k}(\sigma)\right|^{2} d \mathrm{v}_{\Sigma(S)}(\sigma) \\
& \leq h^{N-\tilde{N}} \cdot \sum_{k=0}^{\infty} \frac{\Gamma(2 k+N+n)}{h^{2 k+N+n}} \int_{\Sigma(S)}\left|\varphi_{k}(\sigma)\right|^{2} d \mathrm{v}_{\Sigma(S)}(\sigma) \\
& =h^{N-\tilde{N}} \cdot\|\varphi\|_{(h, N)}^{2} .
\end{aligned}
$$

\section{Comparison of quantization operators}

In this section we compare the two operators $T_{\left(h_{0}, N_{0}\right)}^{S}$ and $T_{\left(h_{1}, N_{1}\right)}^{S}$. First we prove the following.

THEOREM 3.1. Let $h_{0}=h / 2$ and $N_{0}=\frac{1}{2}\{N-n / 2\}$, then the operator

$$
T_{\left(h_{0}, N_{0}\right)}^{S}: \mathcal{H}^{2}\left(\mathbf{X}_{S}, d m_{(h, N)}(z)\right) \rightarrow L_{2}\left(S^{n}\right)
$$

is an isomorphism.

Remark 3.2. We can see that the case constructed by pairing of polarizations in [23] is a special case of Theorem 3.1 where $h=2 \sqrt{2} \pi$ and $N=n / 2-1$.

Let $\mathcal{P}_{k}$ be the space of homogeneous polynomials of degree $k$ on $\mathbb{C}^{n+1}$. Considered as functions on $\mathbf{X}_{S}$, we denote it by $\mathcal{P}_{k}\left(\mathbf{X}_{S}\right)$.

Let $\mathcal{S}_{k}$ be the space of harmonic polynomials of degree $k$ on $\mathbb{R}^{n+1}$. It is well-known that their restrictions to the sphere coincides with an eigenspace of the Laplace operator $\Delta_{S^{n}}$ on the sphere $S^{n}$ having the eigenvalue $\lambda_{k}=k(k+n-1)$ where $k=0,1, \ldots$ The dimension of $\mathcal{S}_{k}$ is given by the formula

$$
\operatorname{dim} \mathcal{S}_{k}=\frac{\Gamma(k+n-1)(2 k+n-1)}{\Gamma(k+1) \cdot \Gamma(n)} .
$$

For each $k \in \mathbb{N}$, let $A_{k}^{S}: \mathcal{S}_{k} \rightarrow \mathcal{P}_{k}$ be the map defined by the integral

$$
A_{k}^{S}(f)(z)=\int_{S^{n}} f(x)\langle x, z\rangle^{k} d \operatorname{vol}_{S}(x),
$$

where $\langle x, z\rangle=\sum_{i=0}^{n} x_{i} z_{i}$. Moreover, for each $k$, let $B_{(h, N ; k)}^{S}$ be the map from $\mathcal{P}_{k}\left(\mathbf{X}_{S}\right)$ to $\mathcal{S}_{k}$ defined by

$$
B_{(h, N ; k)}^{S}(p)(x)=\int_{\mathbf{X}_{S}} p(z)\langle x, \bar{z}\rangle^{k} e^{-h\|z\|}\|z\|^{N} \Omega_{S}(z) .
$$

Remark 3.3. Let $\Delta$ be the Laplacian on $\mathbb{R}^{n+1}$, then we have for each fixed $z \in \mathbf{X}_{S}$ that

$$
\Delta\left(\langle x, \bar{z}\rangle^{k}\right)=0
$$

showing that the polynomial defined by the integral above is harmonic. 
Let $C_{(h, N ; k)}^{S}$ be the map from $\mathcal{P}_{k}\left(\mathbf{X}_{S}\right)$ to $\mathcal{P}_{k}\left(\mathbf{X}_{S}\right)$ defined by the integral

$$
C_{(h, N ; k)}^{S}(p)(\lambda)=\int_{\mathbf{X}_{S}} p(z)\langle\bar{z}, \lambda\rangle^{k} e^{-h\|z\|}\|z\|^{N} \Omega_{S}(z) \quad(N>-n) .
$$

By Schur's lemma we know that the composition $B_{(h, N ; k)}^{S} \circ A_{k}^{S}$ is a constant multiple of the identity operator (we write $a_{k}^{S}(h, N)$ for this constant) and can be expressed as an integral operator:

$$
\left(B_{(h, N ; k)}^{S} \circ A_{k}^{S}\right)(f)(y)=\int_{S^{n}}\left(\int_{\mathbf{X}_{S}}\langle x, z\rangle^{k}\langle\bar{z}, y\rangle^{k} e^{-h\|z\|}\|z\|^{N} \Omega_{S}(z)\right) f(x) d \operatorname{vol}_{S}(x) .
$$

The explicit value of $a_{k}^{S}(h, N)$ can be determined by the relation:

$$
\begin{aligned}
\operatorname{tr} & \left.B_{(h, N ; k)}^{S} \circ A_{k}^{S}\right) \\
& =a_{k}^{S}(h, N) \operatorname{dim} \mathcal{S}_{k} \\
& =\int_{\mathbf{X}_{S}} \int_{S^{n}}|\langle x, z\rangle|^{2 k} d m_{(h, N)}(z) \wedge d \operatorname{vol}_{S}(x) \\
& =\int_{\Sigma(S)} \int_{S^{n}}|\langle x, \sigma\rangle|^{2 k} d \operatorname{vol}_{S}(x) \wedge d v_{\Sigma(S)}(\sigma) \times \int_{0}^{\infty}\|z\|^{2 k+N+n-1} e^{-h\|z\|} d\|z\|,
\end{aligned}
$$

where the integral

$$
\int_{S^{n}}|\langle x, \sigma\rangle|^{2 k} d \operatorname{vol}_{S}(x)
$$

is independent of the variable $\sigma \in \Sigma(S)$ and is given by the formula:

$$
\int_{S^{n}}|\langle x, \sigma\rangle|^{2 k} d \operatorname{vol}_{S}(x)=\operatorname{Vol}\left(S^{n-2}\right) \cdot \frac{\Gamma(k+1)}{2^{k} \cdot \Gamma((n-1) / 2) \Gamma(k+(n+1) / 2)} .
$$

We write

$$
a_{k}^{S}(h, N)=\mathbf{V}_{n}^{S}(h, N) \cdot \frac{\Gamma(k+1)^{2} \Gamma(2 k+N+n)}{2^{k} \cdot h^{2 k}(2 k+n-1) \Gamma(k+n-1) \Gamma(k+(n+1) / 2)},
$$

where the constant

$$
\mathbf{V}_{n}^{S}(h, N)=\frac{\Gamma(n) \operatorname{Vol}(\Sigma(S)) \operatorname{Vol}\left(S^{n-2}\right)}{h^{n+N} \Gamma((n-1) / 2)}
$$

does not depend on the parameter $k$. In the following we do not need the precise value of $\mathbf{V}_{n}^{S}(h, N)$, but we note that

$$
\operatorname{Vol}(\Sigma(S))=\operatorname{Vol}\left(S^{n}\right) \cdot \operatorname{Vol}\left(S^{n-1}(1 / \sqrt{2})\right),
$$

where we write $\operatorname{Vol}\left(S^{n-1}(1 / \sqrt{2})\right)$ for the volume of the $(n-1)$-dimensional sphere with radius $1 / \sqrt{2}$.

Again by Schur's lemma we know that the operator $C_{(h, N ; k)}^{S}$ is a constant multiple of the identity operator with a constant $c_{k}^{S}(h, N)$ given through the relation:

$$
\begin{aligned}
\operatorname{tr}\left(C_{(h, N ; k)}^{S}\right) & =c_{k}^{S}(h, N) \operatorname{dim} \mathcal{S}_{k} \\
& =\int_{\mathbf{X}_{S}}\|\lambda\|^{2 k} e^{-h\|\lambda\|}\|\lambda\|^{N} \Omega_{S}=\operatorname{Vol}(\Sigma(S)) \frac{\Gamma(2 k+N+n)}{h^{2 k+N+n}} .
\end{aligned}
$$


For each fixed parameter $\left(h_{0}, N_{0}\right)$ we denote the restriction of $T_{\left(h_{0}, N_{0}\right)}^{S}$ to $\mathcal{P}_{k}\left(\mathbf{X}_{S}\right)$ by $T_{\left(h_{0}, N_{0} ; k\right)}^{S}$. Then the actions of the orthogonal group on $\mathcal{S}_{k}$ and $\mathbf{X}_{S}$ commute with the operators $T_{\left(h_{0}, N_{0} ; k\right)}^{S}$ and $A_{k}^{S}$. Since the representation of $\mathrm{SO}(n+1)$ in $\mathcal{S}_{k}$ is irreducible and occurs only once in $L_{2}\left(S^{n}\right)$, the restriction $T_{\left(h_{0}, N_{0} ; k\right)}^{S}$ must map $\mathcal{P}_{k}\left(\mathbf{X}_{S}\right) \mathcal{S}_{k}$ and $T_{\left(h_{0}, N_{0} ; k\right)}^{S} \circ A_{k}^{S}$ equals a constant multiple of the identity operator with a constant $b_{k}^{S}\left(h_{0}, N_{0}\right)$. Now, the composition $A_{k}^{S} \circ T_{\left(h_{0}, N_{0} ; k\right)}^{S}$ is given by the integral operator:

$$
A_{k}^{S} \circ T_{\left(h_{0}, N_{0} ; k\right)}^{S}(\varphi)(\lambda)=\int_{\mathbf{X}_{S}}\left\langle\pi_{S} \circ \tau_{S}^{-1}(z), \lambda\right\rangle^{k} \varphi(z) e^{-h_{0}\|z\|}\|z\|^{N_{0}} \Omega_{S}(z) .
$$

The constant $b_{k}^{S}\left(h_{0}, N_{0}\right)$ can be obtained by

$$
\begin{aligned}
b_{k}^{S}\left(h_{0}, N_{0}\right) \operatorname{dim} \mathcal{S}_{k} & =\int_{\mathbf{X}_{S}}\left\langle\pi_{S} \circ \tau_{S}^{-1}(z), z\right\rangle^{k} e^{-h_{0}\|z\|}\|z\|^{N_{0}} \Omega_{S}(z) \\
& =\operatorname{Vol}(\Sigma(S)) \cdot \int_{0}^{\infty}\left(\frac{\|z\|}{\sqrt{2}}\right)^{k} e^{-h_{0}\|z\|}\|z\|^{N_{0}+n-1} d\|z\| \\
& =\operatorname{Vol}(\Sigma(S)) \frac{\Gamma\left(k+N_{0}+n\right)}{(\sqrt{2})^{k} \cdot h_{0}^{k+N_{0}+n}} .
\end{aligned}
$$

Summarizing our results, we have

$$
\begin{aligned}
B_{(h, N ; k)}^{S} \circ A_{k}^{S} & =a_{k}^{S}(h, N) \mathrm{Id}, \\
T_{\left(h_{0}, N_{0} ; k\right)}^{S} \circ A_{k}^{S} & =b_{k}^{S}\left(h_{0}, N_{0}\right) \mathrm{Id}, \\
C_{(h, N ; k)}^{S} & =c_{k}^{S}(h, N) \mathrm{Id}, \\
T_{\left(h_{0}, N_{0} ; k\right)}^{S} & =\frac{b_{k}^{S}\left(h_{0}, N_{0}\right)}{a_{k}^{S}(h, N)} B_{(h, N ; k)}^{S} .
\end{aligned}
$$

Of course these relations are similar to those given in [23] and can be deduced by the facts that the sphere is a symmetric space and the action of the orthogonal group is two-point homogeneous.

The norm of the operator $T_{\left(h_{0}, N_{0} ; k\right)}^{S}$ defined on the space $\mathcal{P}_{k}\left(\mathbf{X}_{S}\right)$ and having values in $\mathcal{S}_{k}$ can be calculated as

$$
\begin{aligned}
\left\|T_{\left(h_{0}, N_{0} ; k\right)}^{S}\right\|^{2} & =\frac{b_{k}^{S}\left(h_{0}, N_{0}\right)^{2}}{a_{k}^{S}(h, N)} \\
& =\text { constant } \times \frac{2^{k} h^{2 k} \Gamma\left(k+N_{0}+n\right)^{2} \Gamma(k+(n+1) / 2)}{2^{k} h_{0}^{2 k} \Gamma(2 k+N+n) \Gamma(k+1)^{2}} \times \frac{1}{\operatorname{dim} \mathcal{S}_{k}} .
\end{aligned}
$$

Proof of Theorem 3.1. If we put $h=2 h_{0}$ and $N=2 N_{0}+n / 2$ and apply Gauss's multiplication formula [1] it follows by arguments similar to those in [23] that

$$
\infty>\lim _{k \rightarrow \infty}\left\|T_{\left(h_{0}, N_{0} ; k\right)}^{S}\right\|>0
$$

and

$$
\infty>\lim _{k \rightarrow \infty}\left\|T_{\left(h_{0}, N_{0} ; k\right)}^{S}{ }^{-1}\right\|>0,
$$

which shows Theorem 3.1. 
Remark 3.4. All of these operators $T_{\left(h_{0}, N_{0}\right)}^{S}: \mathcal{H}^{2}\left(\mathbf{X}_{S}, d m_{\left(2 h_{0}, 2 N_{0}+n / 2\right)}\right) \rightarrow L_{2}\left(S^{n}\right)$ are not unitary, but their restriction to $\mathcal{P}_{k}\left(\mathbf{X}_{S}\right)$ coincides with a constant multiple of a unitary operator.

THEOREM 3.5. Let $N \geq \tilde{N}>-n$, and put $h_{0}=h / 2, \quad N_{0}=1 / 2(N-n / 2)$ and $\tilde{N}_{0}=$ $1 / 2(\tilde{N}-n / 2)$. Then $T_{\left(h_{0}, \tilde{N}_{0}\right)}^{S} \circ T_{\left(h_{0}, N_{0}\right)}^{S}{ }^{-1}: L_{2}\left(S^{n}\right) \rightarrow L_{2}\left(S^{n}\right)$, i.e.

$$
L_{2}\left(S^{n}\right) \stackrel{T_{\left(h_{0}, N_{0}\right)}^{S}{ }^{-1}}{\longrightarrow} \mathcal{H}^{2}\left(\mathbf{X}_{S}, d m_{(h, N)}(z)\right) \hookrightarrow \mathcal{H}^{2}\left(\mathbf{X}_{S}, d m_{(h, \tilde{N})}(z)\right) \stackrel{T_{\left(h_{0}, \tilde{N}_{0}\right)}^{S}}{\longrightarrow} L_{2}\left(S^{n}\right)
$$

is a pseudo-differential operator of order $\frac{1}{2}(\tilde{N}-N)$.

COROLlaRY 3.6. Let $-n<\tilde{N}<N$, then the inclusion map (2.9) is compact.

COROLlaRY 3.7. Let $N-\tilde{N}>2 n$, then the operator $T_{\left(h_{0}, \tilde{N}_{0}\right)}^{S} \circ T_{\left(h_{0}, N_{0}\right)}^{S}{ }^{-1}$ is a trace class operator and the trace is given by

$$
\operatorname{tr}\left(T_{\left(h_{0}, \tilde{N}_{0}\right)}^{S} \circ T_{\left(h_{0}, N_{0}\right)}^{S}{ }^{-1}\right)=(h / 2)^{(N-\tilde{N}) / 2} \sum_{k=0}^{\infty} \frac{\Gamma(k+\tilde{N} / 2+(3 n) / 4)}{\Gamma(k+N / 2+(3 n) / 4)} \operatorname{dim} \mathcal{S}_{k} .
$$

For each $N>\tilde{N}$, the operator $T_{\left(h_{0}, \tilde{N}_{0}\right)}^{S}$ maps the space $\mathcal{H}^{2}\left(\mathbf{X}_{S}, d m_{(h, N)}(z)\right)$ onto the Sobolev space on $S^{n}$ of order $(N-\tilde{N}) / 2$, and we have the following.

COROLLARY 3.8. The projective limits

$$
\lim _{N^{\prime}} \mathcal{H}^{2}\left(\mathbf{X}_{S}, d m_{\left(h, N^{\prime}\right)}(z)\right) \cong \bigcap_{N^{\prime}} \mathcal{H}^{2}\left(\mathbf{X}_{S}, d m_{\left(h, N^{\prime}\right)}(z)\right)
$$

are mapped onto $C^{\infty}\left(S^{n}\right)$ by $T_{\left(h_{0}, N_{0}\right)}^{S}$, where $h_{0}=h / 2$ and $N=2 N_{0}+n / 2>-n$.

To prove Theorem 3.5, we recall a criterion for an operator defined by functional calculus with a positive self-adjoint elliptic operator to be a pseudo-differential operator.

THEOREM 3.9. (Taylor [28]) Let A be a first-order positive elliptic pseudo-differential operator of classical type defined on a closed manifold $M$ and let

$$
A=\int_{0}^{+\infty} \lambda d E_{A}(\lambda)
$$

be the spectral decomposition of $A$ with the spectral measure $\left\{E_{A}(\lambda)\right\}$. Furthermore, let $f \in S_{1,0}^{m}(\mathbb{R})(m \in \mathbb{R})$ be in the symbol class of Hörmander, that is $f$ satisfies

$$
\left|\frac{d^{\ell}}{d t^{\ell}}(f)(t)\right| \leq C_{\ell}(1+|t|)^{m-\ell}
$$

for any $\ell$ with constant $C_{\ell}>0$. Then

$$
f(A)=\int_{0}^{+\infty} f(\lambda) d E_{A}(\lambda)=(2 \pi)^{-1 / 2} \int_{-\infty}^{\infty} \hat{f}(t) e^{\sqrt{-1} t A} d t
$$

is a pseudo-differential operator in the class $L_{1,0}^{m}(M)$, where

$$
\hat{f}(t)=(2 \pi)^{-1 / 2} \int_{-\infty}^{\infty} e^{-\sqrt{-1} t x} f(x) d x
$$

is the Fourier transformation of $f$. 
Proof of Theorem 3.5. Let $\varphi \in \mathcal{S}_{k}$ be a harmonic polynomial, then

$$
\begin{aligned}
T_{\left(h_{0}, \tilde{N}_{0}\right)}^{S} \circ T_{\left(h_{0}, N_{0}\right)}^{-1}(\varphi) & =\frac{b_{k}^{S}\left(h_{0}, \tilde{N}_{0}\right)}{b_{k}^{S}\left(h_{0}, N_{0}\right)} \cdot A_{k}^{S^{-1}} \circ A_{k}^{S}(\varphi) \\
& =\frac{b_{k}^{S}\left(h_{0}, \tilde{N}_{0}\right)}{b_{k}^{S}\left(h_{0}, N_{0}\right)}(\varphi)=h_{0}^{N_{0}-\tilde{N}_{0}} \cdot \frac{\Gamma\left(k+\tilde{N}_{0}+n\right)}{\Gamma\left(k+N_{0}+n\right)}(\varphi) .
\end{aligned}
$$

Now, let $f$ be the smooth function on $\mathbb{R}$ defined for $\lambda \geq 0$ by

$$
f(\lambda)=\frac{\Gamma\left(\sqrt{\lambda^{2}+((n-1) / 2)^{2}}+\tilde{N}_{0}+(n+1) / 2\right)}{\Gamma\left(\sqrt{\lambda^{2}+((n-1) / 2)^{2}}+N_{0}+(n+1) / 2\right)}
$$

and let $f(\lambda)=0$ for $\lambda \ll 0$. Then we have

$$
T_{\left(h_{0}, \tilde{N}_{0}\right)}^{S} \circ T_{\left(h_{0}, N_{0}\right)}^{S}=h_{0}^{N_{0}-\tilde{N}_{0}} \cdot \int_{0}^{\infty} f(\lambda) d \mathbf{E}_{\lambda},
$$

where $\left\{\mathbf{E}_{\lambda}\right\}$ is the spectral measure of the square root of the Laplacian $\sqrt{\Delta_{S^{n}}}$ on the sphere. The square root of the Laplacian is a classical-type pseudo-differential operator of order one, so we have to show that $f(\lambda) \in S_{1,0}^{(\tilde{N}-N) / 2}(\mathbb{R})$. First, we prove the following.

LEMMA 3.10. Let $m \in \mathbb{R}$ and assume that $h(t)$ is the smooth function on $\mathbb{R}$ defined for $t>-m,>-\tilde{m}$ by:

$$
h(t)=\frac{\Gamma(t+\tilde{m})}{\Gamma(t+m)}
$$

and $h(t)=0$ for $t<-m, t<\tilde{m}$. Then $h \in S_{1,0}^{\tilde{m}-m}(\mathbb{R})$.

Proof. By the Stirling formula for sufficiently large $t>0$ we have

$$
\begin{aligned}
\frac{\Gamma(t+\tilde{m})}{\Gamma(t+m)} & =\frac{e^{-(t+\tilde{m})}(t+\tilde{m})^{t+\tilde{m}-(1 / 2)}(1+O(1 /|t+\tilde{m}|)}{e^{-(t+m)}(t+m)^{t+m-(1 / 2)}(1+O(1 /|t+m|)} \\
& =\frac{e^{-\tilde{m}}(t+\tilde{m})^{t+\tilde{m}-(1 / 2)}}{e^{-m}(t+m)^{t+m-(1 / 2)}} \cdot O(1) \\
& =e^{m-\tilde{m}} \cdot t^{\tilde{m}-m} \cdot \frac{(1+\tilde{m} / t)^{t+\tilde{m}-1 / 2}}{(1+m / t)^{t+m-1 / 2}} \cdot O(1) \\
& =O\left(t^{\tilde{m}-m}\right) .
\end{aligned}
$$

In fact, we have

$$
\lim _{t \rightarrow \infty} t^{m-\tilde{m}} \cdot \frac{\Gamma(t+\tilde{m})}{\Gamma(t+m)}=1 .
$$


Moreover,

$$
\begin{aligned}
\frac{d}{d t} \frac{\Gamma(t+\tilde{m})}{\Gamma(t+m)} & =\frac{\Gamma(t+\tilde{m})^{\prime}}{\Gamma(t+\tilde{m})} \frac{\Gamma(t+\tilde{m})}{\Gamma(t+m)}-\frac{\Gamma(t+m)^{\prime}}{\Gamma(t+m)} \frac{\Gamma(t+\tilde{m})}{\Gamma(t+m)} \\
& =\frac{\Gamma(t+\tilde{m})}{\Gamma(t+m)}\left(\frac{\Gamma(t+\tilde{m})^{\prime}}{\Gamma(t+\tilde{m})}-\frac{\Gamma(t+m)^{\prime}}{\Gamma(t+m)}\right) \\
& =\frac{\Gamma(t+\tilde{m})}{\Gamma(t+m)}\left(\sum_{\ell=0}^{\infty}\left(\frac{1}{t+m+\ell}-\frac{1}{t+\tilde{m}+\ell}\right)\right) \\
& =O\left(t^{\tilde{m}-m-1}\right),
\end{aligned}
$$

where we used the formula of the logarithmic derivative of the Gamma function:

$$
\frac{\Gamma(t)^{\prime}}{\Gamma(t)}=-C_{e}+\sum_{\ell=0}^{\infty}\left(\frac{1}{\ell+1}-\frac{1}{t+\ell}\right), \quad C_{e}=\text { Euler constant }
$$

Applying the formula of the higher-order derivative of

$$
\frac{d^{k}}{d t^{k}}\left(\frac{\Gamma(t)^{\prime}}{\Gamma(t)}\right)=\sum_{\ell=0}^{\infty} \frac{(-1)^{k+1} k !}{(\ell+t)^{k+1}}
$$

and by induction we have

$$
\frac{d^{k}}{d t^{k}} h(t)=O\left(t^{\tilde{m}-m-k}\right)
$$

Now, let $r(\lambda)=\sqrt{\lambda^{2}+((n-1) / 2)^{2}}$, then $r(\lambda) \in S_{1,0}^{1}(\mathbb{R})$ and our function $f$ is given by $h(r(\lambda))$ with $\tilde{m}=\tilde{N}_{0}+(n+1) / 2$ and $m=N_{0}+(n+1) / 2$. Finally and by induction again we find that $f(\lambda)=h(r(\lambda)) \in S_{1,0}^{(\tilde{N}-N) / 2}(\mathbb{R})$.

Remark 3.11. So far, we have treated the cases $N>-n$. For general $N$, if we define the norms on the space $\mathcal{P}_{k}\left(\mathbf{X}_{S}\right)$ for $2 k+N+n \leq 0$ in a suitable way, and for $2 k+N+n>0$ by the integral (2.8), then our argument above is valid for such spaces of holomorphic functions on $\mathbf{X}_{S}$.

\section{Reproducing kernel}

In this section we discuss a property of the reproducing kernel $\mathcal{K}_{(h, N)}^{S}$ of the Hilbert space $\mathcal{H}^{2}\left(\mathbf{X}_{S}, d m_{(h, N)}(z)\right)$. First, from the arguments in the preceding section we know that $\mathcal{K}_{(h, N)}^{S}(z, \lambda)$ (we also write $\mathcal{K}^{S}(z, \lambda)$, when the parameters $(h, N)$ are fixed) can be expressed in the form of a power series:

$$
\begin{aligned}
\mathcal{K}^{S}(z, \lambda) & =\sum_{k=0}^{\infty} \frac{1}{c_{k}^{S}(h, N)}\langle z, \bar{\lambda}\rangle^{k} \\
& =\sum_{k=0}^{\infty} \frac{1}{a_{k}^{S}(h, N)} \int_{S^{n}}\langle x, z\rangle^{k}\langle x, \bar{\lambda}\rangle^{k} d \operatorname{vol}_{S}(x) .
\end{aligned}
$$

Note that for each $k$, we have

$$
\frac{1}{a_{k}^{S}(h, N)} \int_{S^{n}}\langle x, z\rangle^{k}\langle x, \bar{\lambda}\rangle^{k} d \operatorname{vol}_{S}(x)=\frac{1}{c_{k}^{S}(h, N)}\langle z, \bar{\lambda}\rangle^{k}
$$


and the series converges on the whole space $\mathbb{C}^{n+1} \times \mathbb{C}^{n+1}$. We remark, that with a function $f \in \mathcal{H}^{2}\left(\mathbf{X}_{S}, d m_{(h, N)}(z)\right)$ Proposition 4.1 below follows from the identity

$$
f(\lambda)=\left\langle f(\bullet), \mathcal{K}^{S}(\bullet, \lambda)\right\rangle_{(h, N)}=\sum_{k=0}^{\infty} \frac{1}{c_{k}^{S}(h, N)} \int_{\mathbf{X}_{S}} f(z)\langle\bar{z}, \lambda\rangle^{k} e^{-h\|z\|}\|z\|^{N} \Omega_{S}(z) .
$$

Proposition 4.1. Every function $f \in \mathcal{H}^{2}\left(\mathbf{X}_{S}, d m_{(h, N)}(z)\right)$ can be extended to the whole complex space $\mathbb{C}^{n+1}$, i.e. the correspondence

$$
\mathcal{H}^{2}\left(\mathbf{X}_{S}, d m_{(h, N)(z)}\right) \ni f \mapsto\left\langle f(\bullet), \mathcal{K}^{S}(\bullet, \lambda)\right\rangle_{(h, N)}
$$

is continuous to the space of all holomorphic functions on $\mathbb{C}^{n+1}$ with the topology of locally uniform convergence.

For (measurable) $f: \mathbb{C}^{n} \rightarrow \mathbb{C}$ and $\|f\|^{2}=\left(1 / \pi^{n}\right) \int_{\mathbb{C}^{n}}|f(z)|^{2} e^{-\|z\|^{2}} \Omega_{\mathbb{R}}$ the SegalBargmann space is defined by

$$
\mathcal{H}^{2}\left(\mathbb{C}^{n}, \pi^{-n} e^{-\|z\|^{2}} \Omega_{\mathbb{R}}\right)=\left\{f \mid f \text { entire function, }\|f\|^{2}<\infty\right\} .
$$

Here $\Omega_{\mathbb{R}}$ denotes the Liouville volume form on $T^{*}\left(\mathbb{R}^{n}\right) \cong \mathbb{C}^{n}$ or the Lebesgue measure on $\mathbb{R}^{2 n}$. In this classical case the corresponding reproducing kernel is well-known to be $\mathcal{K}^{\mathbb{R}}(z, \lambda)=e^{\langle z, \lambda\rangle}$ and the product

$$
\mathcal{K}^{\mathbb{R}}(\lambda, \lambda) e^{-\|\lambda\|^{2}} \Omega_{\mathbb{R}}=\Omega_{\mathbb{R}}
$$

returns to the Lebesgue measure on $\mathbb{R}^{2 n}$.

Corresponding to (4.2) we prove a property for the product of the reproducing kernel $\mathcal{K}_{(h, N)}^{S}(z, \lambda)$ of the Hilbert space $\mathcal{H}^{2}\left(\mathbf{X}_{S}, d m_{(h, N)}(z)\right)$ and the weight function of $d m_{(h, N)}(z)$ with respect to $\Omega_{S}$.

THEOREM 4.2. Let $N>-n$, then

$$
\lim _{\|\lambda\| \rightarrow \infty} \mathcal{K}_{(h, N)}^{S}(\lambda, \lambda) e^{-h\|\lambda\|}\|\lambda\|^{N}=2^{1-n} \frac{h^{n}}{\operatorname{Vol}(\Sigma(S)) \Gamma(n)} .
$$

First, we provide a lemma.

LEMMA 4.3. Let $\left\{a_{k}\right\}$ and $\left\{b_{k}\right\}$ be positive sequences such that

$$
\lim _{k \rightarrow \infty} \frac{b_{k}}{a_{k}}=1,
$$

and the power series $\sum a_{k} x^{k}$ and $\sum b_{k} x^{k}$ converge for all $x \in \mathbb{R}$, then

$$
\lim _{x \rightarrow+\infty} \frac{\sum b_{k} x^{k}}{\sum a_{k} x^{k}}=1
$$

Proof. Let $\epsilon>0$, and let $k_{\epsilon}$ be such that

$$
\left|\frac{b_{k}}{a_{k}}-1\right|<\epsilon, \quad \text { for } k>k_{\epsilon},
$$


i.e. $\left|b_{k}-a_{k}\right|<\epsilon a_{k}$, for $k>k_{\epsilon}$. Then

$$
\begin{aligned}
\left|\frac{\sum_{k=0}^{\infty} b_{k} x^{k}}{\sum_{k=0}^{\infty} a_{k} x^{k}}-1\right| & \leq\left|\frac{\sum_{k \leq k_{\epsilon}}\left(b_{k}-a_{k}\right) x^{k}}{\sum_{k=0}^{\infty} a_{k} x^{k}}\right|+\left|\frac{\sum_{k \geq k_{\epsilon}}\left(b_{k}-a_{k}\right) x^{k}}{\sum_{k=0}^{\infty} a_{k} x^{k}}\right| \\
& <\left|\frac{\sum_{k \leq k_{\epsilon}}\left(b_{k}-a_{k}\right) x^{k}}{\sum_{k=0}^{\infty} a_{k} x^{k}}\right|+\epsilon
\end{aligned}
$$

So if we take $x \gg 0$, then

$$
\left|\frac{\sum_{k=0}^{\infty} b_{k} x^{k}}{\sum_{k=0}^{\infty} a_{k} x^{k}}-1\right|<2 \epsilon
$$

which shows the Lemma.

Proof of Theorem 4.2. We recall that the reproducing kernel of the Hilbert space $\mathcal{H}^{2}\left(\mathbf{X}_{S}, d m_{(h, N)}(z)\right)$ has the form

$$
\mathcal{K}^{S}(z, \lambda)=\sum_{k=0}^{\infty} \frac{\langle z, \bar{\lambda}\rangle^{k}}{c_{k}}
$$

where

$$
c_{k} \operatorname{dim} \mathcal{S}_{k}=\operatorname{Vol}(\Sigma(S)) \frac{\Gamma(2 k+N+n)}{h^{2 k+N+n}}
$$

and

$$
\operatorname{dim} \mathcal{S}_{k}=\frac{\Gamma(k+n-1)(2 k+n-1)}{\Gamma(k+1) \Gamma(n)} .
$$

So

$$
\begin{aligned}
\mathcal{K}^{S}(z, \lambda) & =\sum_{k=0}^{\infty} \frac{h^{n+N}}{\operatorname{Vol}(\Sigma(S)) \Gamma(n)} \frac{\Gamma(k+n-1)(2 k+n-1)}{\Gamma(2 k+N+n) \Gamma(k+1)} \cdot h^{2 k} \cdot\langle z, \bar{\lambda}\rangle^{k} \\
& =C(h, n, N) \sum_{k=0}^{\infty} \frac{\Gamma(k+n-1)(2 k+n-1)}{\Gamma(2 k+N+n) \Gamma(k+1)} \cdot h^{2 k} \cdot\langle z, \bar{\lambda}\rangle^{k},
\end{aligned}
$$

where

$$
C(h, n, N)=\frac{h^{n+N}}{\operatorname{Vol}(\Sigma(S)) \cdot \Gamma(n)} .
$$

In particular, $\mathcal{K}_{S}$ can be expressed on the diagonal of $\mathbf{X}_{S} \times \mathbf{X}_{S}$ in the form

$$
\mathcal{K}^{S}(z, z)=C(h, n, N) \sum_{k=0}^{\infty} \frac{\Gamma(k+n-1)(2 k+n-1)}{\Gamma(2 k+N+n) \Gamma(k+1)} \cdot h^{2 k} \cdot|z|^{2 k},
$$

and we decompose it as

$$
\begin{aligned}
& \frac{\mathcal{K}^{S}(z, z)}{C(h, n, N)} \\
& =\left\{\sum_{k=0}^{\infty} 2 \cdot\|h z\|^{2} \frac{\Gamma(k+n)}{\Gamma(2 k+N+n+2) \Gamma(k+1)} \cdot\|h z\|^{2 k}\right. \\
& \left.\quad+(n-1) \sum_{k=0}^{\infty} \frac{\Gamma(k+n-1)}{\Gamma(2 k+N+n) \Gamma(k+1)} \cdot\|h z\|^{2 k}\right\} .
\end{aligned}
$$


First, let us consider the term (4.4)

$$
\sum_{k=0}^{\infty} 2 \cdot\|h z\|^{2} \frac{\Gamma(k+n)}{\Gamma(2 k+N+n+2) \Gamma(k+1)} \cdot\|h z\|^{2 k},
$$

which we express simply as

$$
2 x^{2} \sum_{k=0}^{\infty} \frac{\Gamma(k+n)}{\Gamma(2 k+N+n+2) \Gamma(k+1)} \cdot x^{2 k} .
$$

We fix $k_{0} \in \mathbb{N}$ in such a way that

$$
2 k_{0}+2+N \geq 0,
$$

and put $\ell=k-k_{0}$, then

$$
\begin{aligned}
\lim _{k \rightarrow \infty} & \frac{\Gamma(k+n)}{\Gamma(2 k+N+n+2) \Gamma(k+1)} \cdot \frac{2^{2 k_{0}+N+n+1} \Gamma(2 \ell+1) \Gamma\left(\ell+2 k_{0}+N+3\right)}{\Gamma(\ell+1)} \\
= & \lim _{\ell \rightarrow \infty} \frac{\Gamma\left(\ell+k_{0}+n\right)}{\Gamma\left(\ell+k_{0}+(N+n+2) / 2\right) \Gamma\left(\ell+k_{0}+(N+n+3) / 2\right) \Gamma\left(\ell+k_{0}+1\right)} \\
& \times \frac{\Gamma(\ell+1 / 2) \Gamma(\ell+1) \Gamma\left(\ell+2 k_{0}+N+3\right)}{\Gamma(\ell+1)}=1 .
\end{aligned}
$$

So, instead of the series

$$
2 x^{2} \sum_{k=0}^{\infty} \frac{\Gamma(k+n)}{\Gamma(2 k+N+n+2) \Gamma(k+1)} \cdot x^{2 k},
$$

we consider

$$
\begin{aligned}
2 x^{2} \sum_{k=0}^{k_{0}-1} & \frac{\Gamma(k+n)}{\Gamma(2 k+N+n+2) \Gamma(k+1)} \cdot x^{2 k} \\
& +\frac{x^{2 k_{0}+2}}{2^{2 k_{0}+N+n}} \sum_{\ell=0}^{\infty} \frac{\Gamma(\ell+1)}{\Gamma\left(\ell+2 k_{0}+N+3\right)} \cdot \frac{x^{2 \ell}}{\Gamma(2 \ell+1)} .
\end{aligned}
$$

The second term can be expressed as

$$
\begin{aligned}
& \frac{x^{2 k_{0}+2}}{2^{2 k_{0}+N+n}} \sum_{\ell=0}^{\infty} \frac{\Gamma(\ell+1)}{\Gamma\left(\ell+2 k_{0}+N+3\right)} \cdot \frac{x^{2 \ell}}{\Gamma(2 \ell+1)} \\
& =\frac{x^{2 k_{0}+2}}{2^{2 k_{0}+N+n} \cdot \Gamma\left(2 k_{0}+N+2\right)} \sum_{\ell=0}^{\infty} \int_{0}^{1} t^{\ell}(1-t)^{2 k_{0}+N+1} d t \frac{x^{2 \ell}}{(2 \ell) !} \\
& =\frac{x^{2 k_{0}+2}}{2^{2 k_{0}+N+n} \cdot \Gamma\left(2 k_{0}+N+2\right)} \int_{0}^{1}(1-t)^{2 k_{0}+N+1} \cosh (\sqrt{t} x) d t
\end{aligned}
$$

Since $2 k_{0}+2+N \geq 0$, the integral

$$
e^{-x} x^{N} \cdot \frac{x^{2 k_{0}+2}}{2^{2 k_{0}+N+n} \cdot \Gamma\left(2 k_{0}+N+2\right)} \cdot \int_{0}^{1}(1-t)^{2 k_{0}+N+1} e^{-\sqrt{t} x} d t
$$


converges to zero when $x \rightarrow \infty$. So we only consider the term

$$
\frac{x^{2 k_{0}+2}}{2^{2 k_{0}+N+n} \Gamma\left(2 k_{0}+N+2\right)} \int_{0}^{1}(1-t)^{2 k_{0}+N+1} \cdot \frac{e^{\sqrt{t} x}}{2} d t .
$$

Now, by the coordinate change $\sqrt{t} x=y$, this integral is equal to

$$
\begin{aligned}
\frac{x^{2 k_{0}+2}}{2^{2 k_{0}+N+n} \cdot \Gamma\left(2 k_{0}+N+2\right)} \int_{0}^{1}(1-t)^{2 k_{0}+N+1} \cdot \frac{e^{\sqrt{t} x}}{2} d t \\
=\frac{x^{2 k_{0}+2}}{2^{2 k_{0}+N+n} \Gamma\left(2 k_{0}+N+2\right)} \int_{0}^{x}\left(1-(y / x)^{2}\right)^{2 k_{0}+N+1} \cdot e^{y} \cdot \frac{y}{x^{2}} d y \\
=\frac{x^{2 k_{0}+2}}{x^{4 k_{0}+2 N+2+2} \cdot 2^{2 k_{0}+N+n} \cdot \Gamma\left(2 k_{0}+N+2\right)} \int_{0}^{x}\left(x^{2}-y^{2}\right)^{2 k_{0}+N+1} \cdot y \cdot e^{y} d y \\
=\frac{x^{2 k_{0}+2} \cdot e^{x}}{x^{4 k_{0}+2 N+2+2} \cdot 2^{2 k_{0}+N+n} \cdot \Gamma\left(2 k_{0}+N+2\right)} \\
\quad \times \int_{0}^{x} y^{2 k_{0}+N+1} \cdot(2 x-y)^{2 k_{0}+N+1} \cdot(x-y) \cdot e^{-y} d y .
\end{aligned}
$$

We have

$$
\begin{aligned}
\lim _{x \rightarrow \infty} e^{-x} x^{N} \frac{x^{2 k_{0}+2}}{2^{2 k_{0}+N+n} \Gamma\left(2 k_{0}+N+2\right)} \int_{0}^{1}(1-t)^{2 k_{0}+N+1} \cosh (\sqrt{t} x) d t \\
=\lim _{x \rightarrow \infty} e^{-x} x^{N}\left\{\frac{x^{2 k_{0}+2} \cdot e^{x}}{x^{4 k_{0}+2 N+2+2} \cdot 2^{2 k_{0}+N+n} \cdot \Gamma\left(2 k_{0}+N+2\right)}\right. \\
\left.\quad \times \int_{0}^{x} y^{2 k_{0}+N+1}(2 x-y)^{2 k_{0}+N+1} \cdot(x-y) \cdot e^{-y} d y\right\} \\
=\lim _{x \rightarrow \infty} x^{N}\left\{\frac{x^{2 k_{0}+2} \cdot x^{2 k_{0}+N+2}}{x^{4 k_{0}+2 N+2+2} \cdot 2^{2 k_{0}+N+n} \cdot \Gamma\left(2 k_{0}+N+2\right)}\right. \\
\left.\quad \times \int_{0}^{x} y^{2 k_{0}+N+1}(2-y / x)^{2 k_{0}+N+1} \cdot(1-y / x) \cdot e^{-y} d y\right\}=2^{1-n} .
\end{aligned}
$$

Of course, we have

$$
\lim _{x \rightarrow \infty} e^{-x} x^{N}\left(2 x^{2} \sum_{k=0}^{k_{0}-1} \frac{\Gamma(k+n)}{\Gamma(2 k+N+n+2) \Gamma(k+1)} \cdot x^{2 k}\right)=0 .
$$

For the second term (4.5) we obtain

$$
\left.K_{2}(h\|z\|)\right)=(n-1) \sum_{k=0}^{\infty} \frac{\Gamma(k+n-1)}{\Gamma(2 k+N+n) \Gamma(k+1)} \cdot\|h z\|^{2 k},
$$

and by replacing $n$ by $n-1$ and $N$ by $N-1$ in the above calculations we find that

$$
e^{-x} x^{N} \cdot K_{2}(x)=O\left(x^{-1}\right) .
$$

Hence, we have proved (4.3). 


\section{Geodesic flow and quantization of a free particle}

In this section we consider the geodesic flow action $\left\{g_{t}\right\}_{t \in \mathbb{R}}$ on the Hilbert space $\mathcal{H}^{2}\left(\mathbf{X}_{S}, d m_{(h, N)}(z)\right)$ and give an aspect of a quantization of a classical free particle. It was described in [23] that the geodesic flow on the sphere is represented simply by the multiplication with the complex number of modulus one $\left\{e^{t \sqrt{-1}}\right\}_{t \in \mathbb{R}}$ through the diffeomorphism $\tau_{S}: T_{0}^{*}\left(S^{n}\right) \rightarrow \mathbf{X}_{S}$. Then for

$$
\varphi(z)=\sum_{k=0}^{\infty} \varphi_{k}(z) \in \mathcal{H}^{2}\left(\mathbf{X}_{S}, d m_{(h, N)}(z)\right)
$$

with homogeneous $\varphi_{k}$ of degree $k$, the action of $g_{t}$ on $\varphi$ is given by

$$
g_{t}: \varphi \mapsto g_{t}(\varphi)(z)=\varphi\left(e^{\sqrt{-1} t} \cdot z\right)=\sum e^{\sqrt{-1} t k} \varphi_{k}(z) .
$$

From this expression we see that the Hilbert space $\mathcal{H}^{2}\left(\mathbf{X}_{S}, d m_{(h, N)}(z)\right)$ is invariant under the geodesic flow action. Let $\left\{e^{\sqrt{-1} t} \sqrt{\Delta_{S^{n}+((n-1) / 2)^{2}}}\right\}_{t \in \mathbb{R}}$ be the one-parameter group of unitary Fourier integral operators generated by the (positive) square root $\sqrt{\Delta_{S^{n}}+((n-1) / 2)^{2}}$ of the Laplace operator. Then, for each $t \in \mathbb{R}$ the canonical relation of the operator $e^{\sqrt{-1} t} \sqrt{\Delta_{S^{n}+((n-1) / 2)^{2}}}$ is the graph of the symplectic isomorphism $g_{t}: T_{0}^{*}\left(S^{n}\right) \rightarrow$ $T_{0}^{*}\left(S^{n}\right)$ (cf. [10], [17] and [28]). In this case the group of symplectic isomorphisms is just $U(1)$, and this gives an example stated in the introduction.

Now, let $N+n>0, h_{0}=h / 2$ and $N_{0}=1 / 2(N-n / 2)$. By Theorem 3.1 we have an isomorphism

$$
T_{\left(h_{0}, N_{0}\right)}^{S}: \mathcal{H}^{2}\left(\mathbf{X}_{S}, d m_{(h, N)}(z)\right) \rightarrow L_{2}\left(S^{n}\right),
$$

as well as two one-parameter groups $\left\{g_{t}\right\}$ and $\left\{e^{\sqrt{-1} t} \sqrt{\Delta_{S^{n}+((n-1) / 2)^{2}}}\right\}$ of unitary operators. Then by comparing both sides of (5.1) below restricted to the eigenspaces of the Laplace operator $\Delta_{S^{n}}$ and after a straightforward calculation one verifies the following.

THEOREM 5.1. We have

$$
T_{\left(h_{0}, N_{0}\right)}^{S} \circ g_{t}=e^{-t \sqrt{-1}(n-1) / 2} e^{\sqrt{-1} t \sqrt{\Delta_{S^{n}+((n-1) / 2)^{2}}}} \circ T_{\left(h_{0}, N_{0}\right)}^{S} .
$$

Since the Hilbert space $\mathcal{H}^{2}\left(\mathbf{X}_{S}, d m_{(h, N)}(z)\right)$ has a reproducing kernel, for each point $z \in \mathbf{X}_{S}$, there is a function $q_{z}(x) \in L_{2}\left(S^{n}\right)$ such that

$$
\begin{aligned}
T_{\left(h_{0}, N_{0}\right)}^{S-1}(f)(z) & =\int_{\mathbf{X}_{S}} T_{\left(h_{0}, N_{0}\right)}^{S-1}(f)(\lambda) \cdot \overline{\mathcal{K}_{(h, N)}^{S}(\lambda, z)} d m_{(h, N)}(\lambda) \\
& =\int_{S^{n}} f(x) \overline{q_{z}(x)} d \operatorname{vol}_{S}(x),
\end{aligned}
$$

and $q_{z}$ is given by

$$
q_{z}(x)=\sum_{k=0}^{\infty} \frac{1}{b_{k}^{S}\left(h_{0}, N_{0}\right)}\langle x, \bar{z}\rangle^{k} .
$$

The function $q_{z}$ can be seen as a quantization of a classical free particle and we have a correspondence of classical and quantum paths.

THEOREM 5.2. We have

$$
q_{e^{\sqrt{-1} t} \cdot z}=e^{t \sqrt{-1}(n-1) / 2} e^{-\sqrt{-1} t \sqrt{\Delta_{S^{n}+((n-1) / 2)^{2}}}}\left(q_{z}\right) .
$$




\section{Complex projective space}

In this section we give a result corresponding to Theorem 3.1 in the case of the complex projective space $P^{n} \mathbb{C}$.

Let $\mathbf{X}_{\mathbb{C}}$ be the subspace in the $(n+1) \times(n+1)$ complex matrices defined by

$$
\mathbf{X}_{\mathbb{C}}=\left\{A \in M(n+1 ; \mathbb{C}) \mid A^{2}=0, \operatorname{rank} A=1\right\} .
$$

In [14] we constructed an isomorphism between $\mathbf{X}_{\mathbb{C}}$ and the punctured (co)tangent bundle $T_{0}^{*}\left(P^{n} \mathbb{C}\right)$ of the $n$-dimensional complex projective space. First, we describe this isomorphism and the complexified Hopf fiber bundle mostly by following [15] and we state our main Theorems 6.8, 6.9 and 7.5 in this and the following sections.

We identify $\mathbb{C}^{n+1} \cong \mathbb{R}^{2 n+2}$ through the correspondence:

$$
\begin{aligned}
\mathbb{C}^{n+1} \ni p & =\left(p_{0}, \ldots, p_{n}\right)=\left(x_{0}+\sqrt{-1} x_{n+1}, \ldots, x_{n}+\sqrt{-1} x_{2 n+1}\right) \\
& =x^{\prime}+\sqrt{-1} x^{\prime \prime} \longleftrightarrow\left(x^{\prime}, x^{\prime \prime}\right) \\
& =\left(x_{0}, x_{1}, \ldots, x_{n}, x_{n+1}, \ldots, x_{2 n+1}\right)=x \in \mathbb{R}^{2 n+2} .
\end{aligned}
$$

$\mathbb{C}^{n+1}$ is equipped with the Hermitian inner product $\langle p, \bar{q}\rangle=\sum p_{i} \bar{q}_{i}$ and the inner product on $\mathbb{R}^{2 n+2}$ is defined by

$$
\operatorname{Re}\langle p, \bar{q}\rangle=\sum_{i=0}^{2 n+1} x_{i} y_{i}=\langle x, y\rangle,
$$

where $p_{i}=x_{i}+\sqrt{-1} x_{n+1+i}, q_{i}=y_{i}+\sqrt{-1} y_{n+1+i}, \bar{q}=\left(\overline{q_{0}}, \ldots, \overline{q_{n}}\right)$.

Let $\pi_{\mathfrak{h}}$ be the projection map (Hopf fibration):

$$
\pi_{\mathfrak{h}}: S^{2 n+1} \longrightarrow P^{n} \mathbb{C} .
$$

We introduce the Riemann metric on $P^{n} \mathbb{C}$ by descending the standard Riemann metric on the unit sphere $S^{2 n+1}=\left\{z \in \mathbb{C}^{n+1} \mid\|z\|=1\right\}$ through $\pi_{\mathfrak{h}}$ and identify the tangent bundle $T\left(P^{n} \mathbb{C}\right)$ and the cotangent bundle $T^{*}\left(P^{n} \mathbb{C}\right)$ by this metric. Then the cotangent bundle $T^{*}\left(P^{n} \mathbb{C}\right) \cong T\left(P^{n} \mathbb{C}\right)$ is realized in the matrix space as follows:

$$
\begin{aligned}
T^{*}\left(P^{n} \mathbb{C}\right) \cong & T\left(P^{n} \mathbb{C}\right) \\
\cong & \{(P, Q) \in M(n+1, \mathbb{C}) \\
& \left.\times M(n+1, \mathbb{C}) \mid P^{2}=P, \operatorname{tr}(P)=1, P Q+Q P=Q, P=P^{*}, Q=Q^{*}\right\},
\end{aligned}
$$

where the inner product on $M(n+1, \mathbb{C})$ is defined by

$$
\operatorname{tr}\left(A \cdot B^{*}\right) \text {. }
$$

We denote the subspace in this space with the condition $Q \neq 0$ by $\mathbf{E}_{\mathbb{C}}$. Recall that

$$
\mathbf{E}_{S} \cong T_{0}\left(S^{2 n+1}\right) \cong T_{0}^{*}\left(S^{2 n+1}\right)
$$

is the punctured (co)tangent bundle of the sphere

$$
\begin{aligned}
T_{0}\left(S^{2 n+1}\right) \cong & \mathbf{E}_{S}=\left\{\left(x^{\prime}, x^{\prime \prime} ; y^{\prime}, y^{\prime \prime}\right) \in \mathbb{R}^{n+1} \times \mathbb{R}^{n+1} \times \mathbb{R}^{n+1} \times \mathbb{R}^{n+1} \mid\left\langle x^{\prime}, x^{\prime}\right\rangle\right. \\
& \left.+\left\langle x^{\prime \prime}, x^{\prime \prime}\right\rangle=1,\left\langle x^{\prime}, y^{\prime}\right\rangle+\left\langle x^{\prime \prime}, y^{\prime \prime}\right\rangle=0, y^{\prime}+\sqrt{-1} y^{\prime \prime}=q \neq 0\right\} .
\end{aligned}
$$


Also, let $\mathbf{E}_{S}^{0}$ be a subspace in $\mathbf{E}_{S}$ such that

$$
\begin{aligned}
\mathbf{E}_{S}^{0}= & \left\{\left(x^{\prime}, x^{\prime \prime} ; y^{\prime}, y^{\prime \prime}\right) \in \mathbb{R}^{4 n+4} \mid\left\langle x^{\prime}, x^{\prime}\right\rangle\right. \\
& \left.+\left\langle x^{\prime \prime}, x^{\prime \prime}\right\rangle=1,\left\langle x^{\prime}, y^{\prime}\right\rangle+\left\langle x^{\prime \prime}, y^{\prime \prime}\right\rangle=0, q+\langle p, \bar{q}\rangle p \neq 0\right\} .
\end{aligned}
$$

The last condition means that the tangent vector

$$
\left(x^{\prime}, x^{\prime \prime} ; y^{\prime}, y^{\prime \prime}\right) \in T_{\left(x^{\prime}, x^{\prime \prime}\right)}\left(S^{2 n+1}\right)
$$

is not parallel to $\left(x^{\prime}, x^{\prime \prime} ;-x^{\prime \prime}, x^{\prime}\right) \in T_{\left(x^{\prime}, x^{\prime \prime}\right)}\left(S^{2 n+1}\right)$. The differential

$$
d \pi_{\mathfrak{h}}: \mathbf{E}_{S}^{0} \rightarrow \mathbf{E}_{\mathbb{C}}
$$

of the map $\pi_{\mathfrak{h}}$ is described as

$$
\begin{gathered}
d \pi_{\mathfrak{h}}\left(x^{\prime}, x^{\prime \prime} ; y^{\prime}, y^{\prime \prime}\right)=d \pi_{\mathfrak{h}}(p, q)=(P, Q), \\
P=\left(p_{i} \overline{p_{j}}\right), \quad Q=\left(q_{i} \bar{p}_{j}\right)+\left(p_{i} \bar{q}_{j}\right), \quad Q \neq 0 .
\end{gathered}
$$

Here again recall that we defined $\tau_{S}\left(x^{\prime}, x^{\prime \prime} ; y^{\prime}, y^{\prime \prime}\right)$ as

$$
\begin{aligned}
\tau_{S}\left(x^{\prime}, x^{\prime \prime} ; y^{\prime}, y^{\prime \prime}\right) & =\left(\|q\| x^{\prime}+\sqrt{-1} y^{\prime},\|q\| x^{\prime \prime}+\sqrt{-1} y^{\prime \prime}\right) \\
& =(u, v) \in \mathbb{C}^{n+1} \times \mathbb{C}^{n+1},
\end{aligned}
$$

and put $\mathbf{X}_{S}^{0}=\tau_{S}\left(\mathbf{E}_{S}^{0}\right)$. Let $\alpha$ be the map

$$
\begin{aligned}
\alpha: \mathbf{X}_{S}^{0} & \longrightarrow \mathbf{X}_{\mathbb{C}}, \\
\mathbf{X}_{S}^{0} \ni(u, v) & \mapsto A=\left(a_{i j}\right), \quad a_{i j}=\left(u_{i}+\sqrt{-1} v_{i}\right)\left(u_{j}-\sqrt{-1} v_{j}\right),
\end{aligned}
$$

then it can be easily seen that $\alpha \circ \tau_{S}\left(x^{\prime}, x^{\prime \prime} ; y^{\prime}, y^{\prime \prime}\right) \in \mathbf{X}_{\mathbb{C}}$. Now, let $\gamma$ be defined by

$$
\begin{aligned}
\gamma: \mathbb{C}^{n+1} \times \mathbb{C}^{n+1} & \longrightarrow \mathbb{C}^{n+1} \times \mathbb{C}^{n+1} \\
(u, v) & \mapsto(z, w), \quad z=u+\sqrt{-1} v, \quad w=u-\sqrt{-1} v,
\end{aligned}
$$

and $\beta: \mathbb{C}^{n+1} \times \mathbb{C}^{n+1} \rightarrow M(n+1, \mathbb{C})$ be the map

$$
\beta(z, w)=\left(a_{i j}\right), \quad a_{i j}=z_{i} w_{j},
$$

then

$$
\gamma\left(\mathbf{X}_{S}^{0}\right)=\left\{(z, w) \in \mathbb{C}^{n+1} \times \mathbb{C}^{n+1} \mid z \neq 0, w \neq 0, B(z, w)=\sum z_{i} w_{i}=0\right\}
$$

and

$$
\beta \circ \gamma=\alpha \text {. }
$$

Through this relation, we return back the obvious $\mathbb{C}^{*}$-action on $\gamma\left(\mathbf{X}_{S}^{0}\right)$ to the space $\mathbf{X}_{S}^{0}$. Then the action is expressed as

$$
\left\{\begin{array}{l}
\mathbf{X}_{S}^{0} \times \mathbb{C}^{*} \rightarrow \mathbf{X}_{S}^{0}, \\
(u, v ; \lambda) \mapsto(u, v) \cdot \lambda=(\tilde{u}, \tilde{v}) \\
\tilde{u}=\frac{1}{2}\left(\lambda+\frac{1}{\lambda}\right) u+\frac{\sqrt{-1}}{2}\left(\lambda-\frac{1}{\lambda}\right) v, \\
\tilde{v}=\frac{-\sqrt{-1}}{2}\left(\lambda-\frac{1}{\lambda}\right) u+\frac{1}{2}\left(\lambda+\frac{1}{\lambda}\right) v .
\end{array}\right.
$$

We make the following remark. 
Proposition 6.1. We remark that $\alpha: \mathbf{X}_{S}^{0} \rightarrow \mathbf{X}_{\mathbb{C}}$ is a $\mathbb{C}^{*}$-principal bundle. We call this the complexified Hopf bundle.

Next, let $\tau_{\mathbb{C}}$ be a map

$$
\begin{gathered}
\tau_{\mathbb{C}}: \mathbf{E}_{\mathbb{C}} \longrightarrow \mathbf{X}_{\mathbb{C}}:(P, Q) \mapsto A \in \mathbf{X}_{\mathbb{C}} \\
A=\|Q\|^{2} P-Q^{2}+\sqrt{\frac{-1}{2}}\|Q\| Q, \quad\|Q\|=\sqrt{\operatorname{tr}\left(Q Q^{*}\right)},
\end{gathered}
$$

and let $\mathbf{E}_{S}^{H}$ be the subspace in $\mathbf{E}_{S}^{0}$ defined by

$$
\mathbf{E}_{S}^{H}=\left\{(p, q) \in \mathbf{E}_{S}^{0} \mid\langle p, \bar{q}\rangle=0\right\} .
$$

Then

$$
d \pi_{\mathfrak{h}}\left(\mathbf{E}_{S}^{H}\right)=\mathbf{E}_{\mathbb{C}}
$$

and the following diagram is commutative:

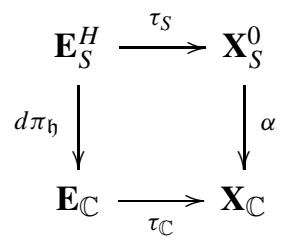

and we know that the map $\tau_{\mathbb{C}}$ is a diffeomorphism between $\mathbf{E}_{\mathbb{C}}$ and $\mathbf{X}_{\mathbb{C}}$.

Note that the diagram

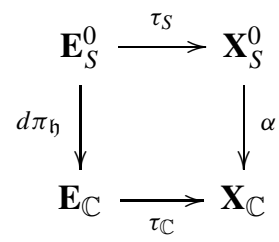

is not commutative. The inverse map $\tau_{\mathbb{C}}^{-1}$ is given as

$$
\begin{gathered}
\tau_{\mathbb{C}}^{-1}(A)=(P, Q), \\
P=\frac{A+A^{*}}{2\|A\|}+\frac{A \cdot A^{*}+A^{*} \cdot A}{2\|A\|^{2}}, \\
Q=\frac{A-A^{*}}{\sqrt{-2 \cdot\|A\|}} .
\end{gathered}
$$

Remark 6.2. Let $(p, q) \in \mathbf{E}_{S}^{H}$, i.e. $(p, q) \in T_{0}\left(S^{2 n+1}\right) \cong \mathbf{E}_{S}$ and assume that $\langle p, \bar{q}\rangle=0$. Then it can be checked for $P=\left(p_{i} \bar{p}_{j}\right), Q=\left(p_{i} \bar{q}_{j}\right)+\left(q_{i} \bar{p}_{j}\right)$ and $A=\tau_{\mathbb{C}}(P, Q)$ that

$$
2\|q\|^{2}=\|Q\|^{2}=\|A\|=\sqrt{\operatorname{tr}\left(A A^{*}\right)} .
$$


PROPOSITION 6.3. The canonical one form $\theta_{\mathbb{C}}$ and the symplectic form $\omega_{\mathbb{C}}$ on the cotangent bundle of the complex projective space are expressed on the space $\mathbf{X}_{\mathbb{C}}$ as follows [14]:

$$
\begin{aligned}
& \theta_{\mathbb{C}}=\sqrt{\frac{-1}{2}}(\partial \sqrt{\|A\|}-\bar{\partial} \sqrt{\|A\|}), \\
& \omega_{\mathbb{C}}=d \theta_{\mathbb{C}}=\sqrt{-2} \bar{\partial} \partial \sqrt{\|A\|} .
\end{aligned}
$$

We use the same notation $\Omega_{\mathbb{C}}$ for the Liouville volume form on $\mathbf{X}_{\mathbb{C}}$ and $\mathbf{E}_{\mathbb{C}}\left(\Omega_{\mathbb{C}}(P, Q)\right.$, or $\left.\Omega_{\mathbb{C}}(A), A \in \mathbf{X}_{\mathbb{C}}\right)$ under the identification $\mathbf{E}_{\mathbb{C}} \stackrel{\tau_{\mathbb{C}}}{\cong} \mathbf{X}_{\mathbb{C}}$. By the decomposition

$$
\begin{gathered}
\mathbf{E}_{\mathbb{C}} \cong \mathbb{R}_{+} \times\left(\mathbf{E}_{\mathbb{C}} \cap\{(P, Q) \mid P, Q \in M(n+1, \mathbb{C}),\|Q\|=1\}\right) \\
\cong \mathbf{X}_{\mathbb{C}} \cong \mathbb{R}_{+} \times\left(\{A \in M(n+1, \mathbb{C}) \mid\|A\|=1\} \cap \mathbf{X}_{\mathbb{C}}\right) \\
=\mathbb{R}_{+} \times \Sigma(\mathbb{C}), \\
\quad(t, P, Q /\|Q\|) \longleftrightarrow(s, A /\|A\|) \in \mathbb{R}_{+} \times \Sigma(\mathbb{C}) \\
s=t^{2}, \quad A=\tau_{\mathbb{C}}(P, Q),
\end{gathered}
$$

we can express $\Omega_{\mathbb{C}}$ as

$$
\Omega_{\mathbb{C}}(A)=\frac{1}{2} s^{n-1} d s \wedge d \mathrm{v}_{\Sigma(\mathbb{C})}(\sigma)
$$

where

$$
A=(\|A\|, A /\|A\|)=(s, \sigma) \in \mathbb{R}_{+} \times \Sigma(\mathbb{C})
$$

and with a (nowhere vanishing) $(4 n-1)$-form $d \mathrm{v}_{\Sigma(\mathbb{C})}(\sigma)$ on

$$
\{A \in M(n+1, \mathbb{C}) \mid\|A\|=1\} \cap \mathbf{X}_{\mathbb{C}} .
$$

Let $\mathcal{P}_{k}=\mathcal{P}_{k}(M(n+1, \mathbb{C}))$ be the space of all homogeneous polynomials on the matrix space $M(n+1, \mathbb{C})$ of degree $k$, and denote their restrictions to $\mathbf{X}_{\mathbb{C}}$ by $\mathcal{P}_{k}\left(\mathbf{X}_{\mathbb{C}}\right)$. For each fixed $(h, N)$ with $h>0$ and $N>-n$ let

$$
d m_{(h, N)}(A)=e^{-h \sqrt{\|A\|}}\|A\|^{N} \Omega_{\mathbb{C}}(A)
$$

be a volume form on the space $\mathbf{X}_{\mathbb{C}}$. We define an inner product on $\sum \oplus \mathcal{P}_{k}\left(\mathbf{X}_{\mathbb{C}}\right)$ by the integral

$$
\langle\varphi, \bar{\psi}\rangle_{(h, N)}=\int_{\mathbf{X}_{\mathbb{C}}} \varphi(A) \overline{\psi(A)} d m_{(h, N)}(A) .
$$

Then for each $k \neq \ell$, the spaces $\mathcal{P}_{k}\left(\mathbf{X}_{\mathbb{C}}\right)$ and $\mathcal{P}_{\ell}\left(\mathbf{X}_{\mathbb{C}}\right)$ are orthogonal. We denote the completion of $\sum \oplus \mathcal{P}_{k}\left(\mathbf{X}_{\mathbb{C}}\right)$ with respect to the norm $\|\bullet\|_{(h, N)}$ defined by this inner product by

$$
\mathcal{H}^{2}\left(\mathbf{X}_{\mathbb{C}}, d m_{(h, N)}(A)\right) .
$$

Remark 6.4. We can prove along the same line as the proof of Proposition 2.4 that there are no bounded holomorphic functions on $\mathbf{X}_{\mathbb{C}}(n \geq 1)$.

Proposition 6.5. Any $\varphi(A) \in \mathcal{H}^{2}\left(\mathbf{X}_{\mathbb{C}}, d m_{(h, N)}(A)\right)$ has an expansion of the form $\varphi(A)=$ $\sum_{k=0}^{\infty} \varphi_{k}(A)$ with kth-order homogeneous polynomials $\varphi_{k}(A)$ on $M(n+1, \mathbb{C})$. 
The norm of $\varphi(A)=\sum_{k=0}^{\infty} \varphi_{k}(A) \in \mathcal{H}^{2}\left(\mathbf{X}_{\mathbb{C}}, d m_{(h, N)}(A)\right)$ can be expressed as

$$
\begin{aligned}
\|\varphi\|_{(h, N)}^{2} & =\int_{\mathbf{X}_{\mathbb{C}}}|\varphi(A)|^{2} d m_{(h, N)}(A) \\
& =\sum_{k=0}^{\infty} \int_{\Sigma(\mathbb{C})}\left|\varphi_{k}(\sigma)\right|^{2} d \mathrm{v}_{\Sigma(\mathbb{C})}(\sigma) \times \int_{0}^{\infty} \frac{1}{2} \cdot\|A\|^{2 k+N+n-1} e^{-h \sqrt{\|A\|}} d\|A\| \\
& =\sum_{k=0}^{\infty} \frac{\Gamma(4 k+2 N+2 n)}{h^{4 k+2 N+2 n}} \int_{\Sigma(\mathbb{C})}\left|\varphi_{k}(\sigma)\right|^{2} d \mathrm{v}_{\Sigma(\mathbb{C})}(\sigma) .
\end{aligned}
$$

Proposition 6.6. Let $\tilde{N} \leq N$, then

$$
\mathcal{H}^{2}\left(\mathbf{X}_{\mathbb{C}}, d m_{(h, N)}(A)\right) \hookrightarrow \mathcal{H}^{2}\left(\mathbf{X}_{\mathbb{C}}, d m_{(h, \tilde{N})}(A)\right)
$$

and the inclusion map is continuous.

Proof. For $\psi \in \mathcal{H}^{2}\left(\mathbf{X}_{\mathbb{C}}, d m_{(h, N)}(A)\right)$, we have

$$
\begin{aligned}
& \sum_{k=0}^{\infty} \frac{\Gamma(4 k+2 \tilde{N}+2 n)}{h^{4 k+2 \tilde{N}+2 n}} \int_{\Sigma(\mathbb{C})}\left|\varphi_{k}(\sigma)\right|^{2} d \mathrm{v}_{\Sigma(\mathbb{C})}(\sigma) \\
& \quad \leq h^{2(N-\tilde{N})} \cdot \sum_{k=0}^{\infty} \frac{\Gamma(4 k+2 N+2 n)}{h^{4 k+2 N+2 n}} \int_{\Sigma(\mathbb{C})}\left|\varphi_{k}(\sigma)\right|^{2} d \mathrm{v}_{\Sigma(\mathbb{C})}(\sigma),
\end{aligned}
$$

and this inequality shows the assertion.

For any positive integer $k \geq 0$, let $\mathcal{S}_{2 k}^{\mathbb{C}}$ be the space of harmonic polynomials on $\mathbb{R}^{2 n+2} \cong$ $\mathbb{C}^{n+1}$ which are invariant under the action of $U(1)$ (and necessarily of even degree), that is, harmonic polynomials of the variables $p_{i} \bar{p}_{j}$. We denote the space of their descents to the complex projective space by $E_{k}^{\mathbb{C}}$.

The proof of Proposition 6.7 below can be found in [6] (also see [7]).

Proposition 6.7. For all $k \in \mathbb{N}_{0}$ the space $E_{k}^{\mathbb{C}}$ is an eigenspace of the Laplacian $\Delta$ on $P^{n} \mathbb{C}$ with the eigenvalue

$$
\lambda_{k}=4 k(n+k) .
$$

Moreover, it holds that $\operatorname{dim} E_{0}^{\mathbb{C}}=1$ and in the case $k \in \mathbb{N}$ one has

$$
\begin{aligned}
\operatorname{dim} E_{k}^{\mathbb{C}} & =\left(\begin{array}{c}
n+k \\
k
\end{array}\right)^{2}-\left(\begin{array}{c}
n+k-1 \\
k-1
\end{array}\right)^{2} \\
& =n(n+2 k) \frac{\Gamma(n+k)^{2}}{\Gamma(n)^{2} \Gamma(k+1)^{2}} .
\end{aligned}
$$

Next, we define an operator $T_{(h, N)}^{\mathbb{C}}$ from functions on $\mathbf{X}_{\mathbb{C}}$ with a suitable integrability condition to functions on $P^{n} \mathbb{C}$.

For a function $\psi(A)$ on $\mathbf{X}_{\mathbb{C}}$, we denote the distribution on $P^{n} \mathbb{C}$

$$
C^{\infty}\left(P^{n} \mathbb{C}\right) \ni f \mapsto \int_{\mathbf{X}_{\mathbb{C}}}\left(\pi_{\mathbb{C}} \circ \tau_{\mathbb{C}}^{-1}\right)^{*}(f)(A) \cdot \psi(A) e^{-h \sqrt{\|A\|}}\|A\|^{N} \Omega_{\mathbb{C}}(A)
$$


by

$$
\left(\pi_{\mathbb{C}} \circ \tau_{\mathbb{C}}^{-1}\right)_{*}\left(\psi(\bullet) \cdot e^{-h \sqrt{\|\bullet\|}}\|\bullet\|^{N} \Omega_{\mathbb{C}}(\bullet)\right)
$$

i.e. a distribution defined by the fiber integration of a $4 n$-degree form

$$
\psi(A) \cdot e^{-h \sqrt{\|A\|}}\|A\|^{N} \Omega_{\mathbb{C}}(A)
$$

along the fiber of the map $\pi_{\mathbb{C}} \circ \tau_{\mathbb{C}}^{-1}: \mathbf{X}_{\mathbb{C}} \rightarrow P^{n} \mathbb{C}$. The resulting form is a smooth $2 n$-degree form on $P^{n} \mathbb{C}$. We denote this form by

$$
T_{(h, N)}^{\mathbb{C}}(\psi)(P) d \operatorname{vol}_{\mathbb{C}}(P),
$$

where $d \operatorname{vol}_{\mathbb{C}}(P)$ is the Riemann volume form on $P^{n} \mathbb{C}$. So we define an operator

$$
T_{(h, N)}^{\mathbb{C}}: \sum \oplus \mathcal{P}_{k}\left(\mathbf{X}_{\mathbb{C}}\right) \longrightarrow C^{\infty}\left(P^{n} \mathbb{C}\right), \quad \psi \mapsto T_{(h, N)}^{\mathbb{C}}(\psi),
$$

and consider its extension to $\mathcal{H}^{2}\left(\mathbf{X}_{\mathbb{C}}, d m_{\left(h^{\prime}, N^{\prime}\right)}(A)\right)$.

THEOREM 6.8. Let $2 h_{0}=h$ and $2 N_{0}+n / 2=N>-n$, then

$$
T_{\left(h_{0}, N_{0}\right)}^{\mathbb{C}}: \mathcal{H}^{2}\left(\mathbf{X}_{\mathbb{C}}, d m_{(h, N)}\right) \longrightarrow L_{2}\left(P^{n} \mathbb{C}\right)
$$

is an isomorphism (but not unitary).

THeOREM 6.9. Let $-n<\tilde{N} \leq N$ and $N_{0}=(N-n / 2) / 2, \tilde{N}_{0}=(\tilde{N}-n / 2) / 2$, then

$$
T_{\left(h / 2, \tilde{N}_{0}\right)}^{\mathbb{C}} \circ\left(T_{\left(h / 2, N_{0}\right)}^{\mathbb{C}}\right)^{-1}: L_{2}\left(P^{n} \mathbb{C}\right) \longrightarrow L_{2}\left(P^{n} \mathbb{C}\right),
$$

is a pseudo-differential operator in $L_{1,0}^{\tilde{N}-N}\left(P^{n} \mathbb{C}\right)$, i.e.

$$
L_{2}\left(P^{n} \mathbb{C}\right) \stackrel{\left(T_{\left(h / 2, N_{0}\right)}^{\mathbb{C}}\right)^{-1}}{\longrightarrow} \mathcal{H}^{2}\left(\mathbf{X}_{\mathbb{C}}, d m_{(h, N)}(A)\right) \hookrightarrow \mathcal{H}^{2}\left(\mathbf{X}_{\mathbb{C}}, d m_{(h, \tilde{N})}(A)\right) \stackrel{T_{\left(h / 2, \tilde{N}_{0}\right)}^{\mathbb{C}}}{\longrightarrow} L_{2}\left(P^{n} \mathbb{C}\right)
$$

is a pseudo-differential operator of order $\tilde{N}-N$ on the complex projective space.

COROllaRY 6.10. For $-n<\tilde{N}<N$ the inclusion map (6.10) is compact.

Remark 6.11. We have introduced a family of volume forms $d m_{(h, N)}(A)$ on $\mathbf{X}_{\mathbb{C}}$ with parameter $(h, N)$ as we did for the sphere case. The form of the weight function is taken from [15] (see also [11]) where it was determined by means of the pairing of polarizations on $\mathbf{X}_{\mathbb{C}}$ (vertical polarization on $\mathbf{X}_{\mathbb{C}}$ and the Kähler polarization given by the realization of the punctured cotangent bundle $T_{0}^{*}\left(P^{n} \mathbb{C}\right)$ as the space $\left.\mathbf{X}_{\mathbb{C}}\right)$. Here we allow, as for the case of the sphere, all positive values of $h$ and we assume that the exponent $N$ fulfills $N>-n$. The proof of Theorem 6.8 is worked along the same lines as given in [23], [11] and [15]. 


\section{Proofs of Theorems 6.8, 6.9 and reproducing kernel}

We define operators $A_{k}^{\mathbb{C}}: E_{k}^{\mathbb{C}} \rightarrow \mathcal{P}_{k}, B_{(h, N ; k)}^{\mathbb{C}}: \mathcal{P}_{k}\left(\mathbf{X}_{\mathbb{C}}\right) \rightarrow E_{k}^{\mathbb{C}}$ and $C_{(h, N ; k)}^{\mathbb{C}}: \mathcal{P}_{k}\left(\mathbf{X}_{\mathbb{C}}\right) \rightarrow$ $\mathcal{P}_{k}\left(\mathbf{X}_{\mathbb{C}}\right)$ as follows:

$$
\begin{aligned}
A_{k}^{\mathbb{C}} & : E_{k}^{\mathbb{C}} \longrightarrow \mathcal{P}_{k}, \\
f & : \mapsto A_{k}^{\mathbb{C}}(f)(A)=\int_{P^{n} \mathbb{C}} f(P)(\operatorname{tr}(P \cdot A))^{k} d \operatorname{vol}_{\mathbb{C}}(P), \\
B_{(h, N ; k)}^{\mathbb{C}} & : \mathcal{P}_{k}\left(\mathbf{X}_{\mathbb{C}} \longrightarrow E_{k}^{\mathbb{C}},\right. \\
\psi & : \mapsto B_{(h, N ; k)}^{\mathbb{C}}(\psi)(P)=\int_{\mathbf{X}_{\mathbb{C}}} \psi(A)\left(\operatorname{tr}\left(P \cdot A^{*}\right)\right)^{k} e^{-h \sqrt{\|A\|}\|A\|^{N} \Omega_{\mathbb{C}}(A),} \\
C_{(h, N ; k)}^{\mathbb{C}}: & \mathcal{P}_{k}\left(\mathbf{X}_{\mathbb{C}}\right) \longrightarrow \mathcal{P}_{k}\left(\mathbf{X}_{\mathbb{C}}\right), \\
\psi & : \mapsto C_{(h, N ; k)}^{\mathbb{C}}(\psi)(A)=\int_{\mathbf{X}_{\mathbb{C}}} \psi(B)\left(\operatorname{tr}\left(A \cdot B^{*}\right)\right)^{k} e^{-h \sqrt{\|B\|}\|B\|^{N} \Omega_{\mathbb{C}}(B) .}
\end{aligned}
$$

It can be easily seen that the function $A_{k}^{\mathbb{C}}(f)$ is a homogeneous polynomial on the matrix space $M(n+1, \mathbb{C})$ and $B_{(h, N ; k)}^{\mathbb{C}}(\psi)(P)$ is a polynomial of variables $p_{i}, \bar{p}_{j} \in \mathbb{C}^{n+1}$ if we put $P=\left(p_{i} \bar{p}_{j}\right)$. Furthermore, $C_{(h, N ; k)}^{\mathbb{C}}(\psi)(A)$ is a polynomial of the variable $A \in M(n+1, \mathbb{C})$ and all of these operators commute with the induced action on the function spaces from the actions of the isometry group $U(n+1)$ on $P^{n} \mathbb{C}$ and the lifted action of $U(n+1)$ to $\mathbf{X}_{\mathbb{C}}$. We note that $B_{(h, N ; k)}^{\mathbb{C}}(\psi) \in E_{k}^{\mathbb{C}}$.

Lemma 7.1. Let $A=\left(a_{i j}\right) \in \mathbf{X}_{\mathbb{C}}$ and let $\psi(A) \in \mathcal{P}_{k}$, then the function

$$
\int_{\mathbf{X}_{\mathbb{C}}} \psi(A)\left(\operatorname{tr}\left(p_{i} \bar{p}_{j}\right) A\right)^{k} e^{-h \sqrt{\|A\|}}\|A\|^{N} \Omega_{\mathbb{C}}(A)
$$

of the variables $p_{i}$ and $\bar{p}_{j}$ is a $U(1)$-invariant harmonic polynomial on $\mathbb{R}^{2 n+2}$.

Proof. The $U(1)$-invariance clearly holds and we note that

$$
\mathbf{X}_{\mathbb{C}}^{*}=\left\{A^{*} \mid A \in \mathbf{X}_{\mathbb{C}}\right\}=\mathbf{X}_{\mathbb{C}} .
$$

The Laplacian $\Delta$ on $\mathbb{C}^{n+1} \cong \mathbb{R}^{2 n+2}$ is equal to $\frac{1}{4} \sum\left(\partial^{2} / \partial p_{i} \partial \bar{p}_{i}\right)$. For $P=\left(p_{i} \bar{p}_{j}\right)$ where $p \in \mathbb{C}^{n+1}$

$$
\begin{aligned}
\left(\Delta B_{(h, N ; k)}^{\mathbb{C}}(\psi)\right)\left(p_{i} \bar{p}_{j}\right)= & \frac{1}{4} \int_{\mathbf{X}_{\mathbb{C}}} \psi(A) \cdot k \cdot\left(\operatorname{tr}\left(p_{i} \bar{p}_{j}\right) A\right)^{k-1} \cdot \operatorname{tr}(A) \cdot e^{-h \sqrt{\|A\|}\|A\|^{N} \Omega_{\mathbb{C}}(A)} \\
& +\frac{1}{4} \int_{\mathbf{X}_{\mathbb{C}}} \psi(A) \cdot k(k-1) \cdot\left(\operatorname{tr}\left(p_{i} \bar{p}_{j}\right) A\right)^{k-2} \\
& \times \sum_{\ell} \sum_{i} p_{i} a_{\ell i} \cdot \sum_{\ell} \sum_{j} \bar{p}_{j} a_{j \ell} \cdot e^{-h \sqrt{\|A\|}\|A\|^{N} \Omega_{\mathbb{C}}(A) .}
\end{aligned}
$$

By $\operatorname{tr}(A)=0$ and

$$
\sum_{\ell} \sum_{i} p_{i} a_{\ell i} \cdot \sum_{\ell} \sum_{j} \bar{p}_{j} a_{\ell j}=\sum_{i j} \sum_{\ell} a_{j \ell} a_{\ell i} p_{i} \bar{p}_{j}=0,
$$


we have

$$
\left(\Delta B_{(h, N ; k)}^{\mathbb{C}}(\psi)\right)\left(p_{i} \bar{p}_{j}\right)=0 .
$$

Let $T_{(h, N ; k)}^{\mathbb{C}}$ denote the restriction of the operator $T_{(h, N)}^{\mathbb{C}}$ to the space $\mathcal{P}_{k}\left(\mathbf{X}_{\mathbb{C}}\right)$, then by Schur's lemma we have the following.

PROPOSITION 7.2. With the constants $a_{k}^{\mathbb{C}}(h, N)>0$ and $b_{k}^{\mathbb{C}}(h, N)>0$ we have

$$
B_{(h, N ; k)}^{\mathbb{C}} \circ A_{k}^{\mathbb{C}}=a_{k}^{\mathbb{C}}(h, N) \mathrm{Id},
$$

and

$$
T_{(h, N ; k)}^{\mathbb{C}} \circ A_{k}^{\mathbb{C}}=b_{k}^{\mathbb{C}}(h, N) \text { Id. }
$$

Proof. We only remark that the image $T_{(h, N ; k)}^{\mathbb{C}} \circ A_{k}^{\mathbb{C}}\left(E_{k}^{\mathbb{C}}\right)$ must be in $E_{k}^{\mathbb{C}}$, since $P^{n} \mathbb{C}$ is a compact symmetric space, so that an irreducible representation can occur only once in $L_{2}\left(P^{n} \mathbb{C}\right)$.

By the proposition above, the space $\mathcal{P}_{k}\left(\mathbf{X}_{\mathbb{C}}\right)$ is also an irreducible representation space of $U(n+1)$, so we have the following.

PROPOSITION 7.3. The operator $C_{(h, N ; k)}^{\mathbb{C}}$ is a constant multiple of the identity operator with a constant $c_{k}^{\mathbb{C}}(h, N)$.

Now, we determine the constants $a_{k}^{\mathbb{C}}(h, N), b_{k}^{\mathbb{C}}(h, N)$ and $c_{k}^{\mathbb{C}}(h, N)$. Since the kernel of the operator $B_{(h, N ; k)}^{\mathbb{C}} \circ A_{k}^{\mathbb{C}}$ is

$$
\int_{\mathbf{X}_{\mathbb{C}}}(\operatorname{tr}(P \cdot A))^{k} \cdot\left(\operatorname{tr}\left(P^{\prime} \cdot A^{*}\right)\right)^{k} \cdot e^{-h \sqrt{\|A\|}}\|A\|^{N} \Omega_{\mathbb{C}}(A)
$$

we have

$$
\begin{aligned}
& a_{k}^{\mathbb{C}}(h, N) \cdot \operatorname{dim} E_{k}^{\mathbb{C}} \\
& \quad=\int_{P^{n} \mathbb{C}} \int_{\mathbf{X}_{\mathbb{C}}}(\operatorname{tr}(P \cdot A))^{k} \cdot\left(\operatorname{tr}\left(P \cdot A^{*}\right)\right)^{k} \cdot e^{-h \sqrt{\|A\|}\|A\|^{N} \Omega_{\mathbb{C}}(A) \wedge d \operatorname{vol}_{\mathbb{C}}(P)} \\
& \quad=\int_{P^{n} \mathbb{C}} \int_{\mathbf{X}_{\mathbb{C}}}|(\operatorname{tr}(P \cdot A))|^{2 k} e^{-h \sqrt{\|A\|}}\|A\|^{N} \Omega_{\mathbb{C}}(A) \wedge d \operatorname{vol}_{\mathbb{C}}(P) .
\end{aligned}
$$

Here, by the two-point homogeneity of the action of the unitary group $U(n+1)$ on $P^{n} \mathbb{C}$, the first integral

$$
\int_{\mathbf{X}_{\mathbb{C}}}|(\operatorname{tr}(P \cdot A))|^{2 k} e^{-h \sqrt{\|A\|}}\|A\|^{N} \Omega_{\mathbb{C}}(A)
$$

in (7.3) does not depend on $A /\|A\|$. So we can put

$$
A=\left(\begin{array}{cccc}
0 & 1 & \cdots & 0 \\
0 & 0 & \cdots & 0 \\
\vdots & \vdots & \ddots & \vdots \\
0 & 0 & \cdots & 0
\end{array}\right)
$$


and the integral (7.3) is equal to

$$
\begin{aligned}
a_{k}^{\mathbb{C}}(h, N) \cdot \operatorname{dim} E_{k}^{\mathbb{C}}= & \int_{P^{n} \mathbb{C}} \int_{\mathbf{X}_{\mathbb{C}}}\left(x_{0}^{2}+x_{n+1}^{2}\right)^{k}\left(x_{1}^{2}+x_{n+2}^{2}\right)^{k} \\
& \times\|A\|^{2 k} e^{-h \sqrt{\|A\|}}\|A\|^{N} \Omega_{\mathbb{C}}(A) \wedge d \operatorname{vol}_{\mathbb{C}}(P)=(*) .
\end{aligned}
$$

Now, by the relation

$$
d \operatorname{vol}_{\mathbb{C}}=\frac{1}{2 \pi}\left(\pi_{\mathfrak{h}}\right)_{*}\left(d \operatorname{vol}_{S}\right),
$$

the last integral is given by

$$
\begin{aligned}
(*)= & \frac{1}{2 \pi} \int_{S^{2 n+1}}\left(x_{0}^{2}+x_{n+1}^{2}\right)^{k}\left(x_{1}^{2}+x_{n+2}^{2}\right)^{k} d \operatorname{vol}_{S}(x) \cdot \int_{\mathbf{X}_{\mathbb{C}}} e^{-h \sqrt{\|A\|}}\|A\|^{N+2 k} \Omega_{\mathbb{C}}(A) \\
= & \frac{1}{2 \pi} \int_{S^{2 n+1}}\left(x_{0}^{2}+x_{n+1}^{2}\right)^{k}\left(x_{1}^{2}+x_{n+2}^{2}\right)^{k} d \operatorname{vol}_{S}(x) \\
& \times \int_{0}^{\infty} e^{-h t} t^{2 N+4 k+2 n-1} d t \cdot \int_{\Sigma(\mathbb{C})} d \mathrm{v}_{\Sigma(\mathbb{C})} \\
= & \frac{\pi}{2} \frac{\Gamma(n-1) \Gamma(k+1)^{2}}{\Gamma(2 k+n+1)} \operatorname{Vol}\left(S^{2 n-3}\right) \cdot \frac{\Gamma(2 N+4 k+2 n)}{h^{2 N+4 k+2 n}} \cdot \operatorname{Vol}(\Sigma(\mathbb{C})) .
\end{aligned}
$$

The integral

$$
I_{k}=\int_{S^{2 n+1}}\left(x_{0}^{2}+x_{n+1}^{2}\right)^{k}\left(x_{1}^{2}+x_{n+2}^{2}\right)^{k} d \operatorname{vol}_{S}(x)
$$

is calculated as

$$
I_{k}=\pi^{2} \frac{\Gamma(n-1) \Gamma(k+1)^{2}}{\Gamma(2 k+n+1)} \cdot \operatorname{Vol}\left(S^{2 n-3}\right) \quad(n>1)
$$

and we do not present the details here (see [23, Appendix]).

We determine the constant $b_{k}^{\mathbb{C}}(h, N)$. The kernel of $A_{k}^{\mathbb{C}} \circ T_{(h, N ; k)}^{\mathbb{C}}$ is given by

$$
\left(\operatorname{tr}\left(\pi_{\mathbb{C}} \circ \tau_{\mathbb{C}}^{-1}(B) \cdot A\right)\right)^{k} e^{-h \sqrt{\|A\|}}\|A\|^{N}
$$

so

$$
\begin{aligned}
b_{k}^{\mathbb{C}}(h, N) \operatorname{dim} E_{k}^{\mathbb{C}} & =\int_{\mathbf{X}_{\mathbb{C}}}\left(\operatorname{tr}\left(\pi_{\mathbb{C}} \circ \tau_{\mathbb{C}}^{-1}(A) \cdot A\right)\right)^{k} e^{-h \sqrt{\|A\|}}\|A\|^{N} \Omega_{\mathbb{C}}(A) \\
& =\int_{\mathbf{X}_{\mathbb{C}}}\left(\frac{1}{2}\|A\|\right)^{k} e^{-h \sqrt{\|A\|}\|A\|^{N} \Omega_{\mathbb{C}}(A)} \\
& =\frac{\Gamma(2 N+2 k+2 n)}{2^{k} h^{2 N+2 k+2 n}} \cdot \operatorname{Vol}(\Sigma(\mathbb{C})) .
\end{aligned}
$$

The constant $c_{k}^{\mathbb{C}}(h, N)$ is calculated as

$$
\begin{aligned}
c_{k}^{\mathbb{C}}(h, N) \operatorname{dim} E_{k}^{\mathbb{C}} & =\int_{\mathbf{X}_{\mathbb{C}}}\left(\operatorname{tr}\left(A \cdot A^{*}\right)\right)^{k} e^{-h \sqrt{\|A\|}}\|A\|^{N} \Omega_{\mathbb{C}}(A) \\
& =\operatorname{Vol}(\Sigma(\mathbb{C})) \cdot \frac{\Gamma(2 N+4 k+2 n)}{h^{2 N+4 k+2 n}} .
\end{aligned}
$$


Proof of Theorem 6.8. Since

$$
\begin{aligned}
& \int_{\mathbf{X}_{\mathbb{C}}}\left|A_{k}^{\mathbb{C}}(f)(A)\right|^{2} d m_{(h, N)}(A) \\
& \quad=\int_{P^{n} \mathbb{C}} B_{(h, N ; k)}^{\mathbb{C}}\left(A_{k}^{\mathbb{C}}(f)\right)(P) \cdot \overline{f(P)} d \operatorname{vol}_{\mathbb{C}}(P)=a_{k}^{\mathbb{C}}(h, N)\|f\|^{2},
\end{aligned}
$$

the norm of the operator $T_{(\tilde{h}, \tilde{N} ; k)}^{\mathbb{C}}$ is given by

$$
\left\|T_{\tilde{h}, \tilde{N} ; k)}^{\mathbb{C}}\right\|^{2}=\frac{b_{k}^{\mathbb{C}}(\tilde{h}, \tilde{N})^{2}}{a_{k}^{\mathbb{C}}(h, N)} .
$$

Note that $\overline{\operatorname{tr}(P A)}=\operatorname{tr}\left(P A^{*}\right)$ in the above calculation. Then, by the explicit data of the constants $b_{k}^{\mathbb{C}}(\tilde{h}, \tilde{N})$ and $a_{k}^{\mathbb{C}}(h, N)$ we have for $k \in \mathbb{N}$

$$
\begin{aligned}
\frac{b_{k}^{\mathbb{C}}(\tilde{h}, \tilde{N})^{2}}{a_{k}^{\mathbb{C}}(h, N)}= & \frac{1}{\operatorname{dim} E_{k}^{\mathbb{C}}} \cdot\left(\frac{\operatorname{Vol}(\Sigma(\mathbb{C}))}{\tilde{h}^{2 N+2 n}} \frac{\Gamma(2 k+2 \tilde{N}+2 n)}{2^{k} \tilde{h}^{2 k}}\right)^{2} \\
& \times \frac{2 h^{2 N+2 n}}{\pi \cdot \operatorname{Vol}(\Sigma(\mathbb{C})) \operatorname{Vol}\left(S^{2 n-3}\right) \Gamma(n-1) \Gamma(k+1)^{2}} \frac{h^{4 k} \Gamma(2 k+n+1)}{\Gamma(4 k+2 N+2 n)} \\
= & C(h, \tilde{h}, N, \tilde{N}, n) \cdot \frac{1}{\Gamma(n+k)^{2}} \cdot \frac{2^{4 k} \cdot \Gamma(k+\tilde{N}+n)^{2} \Gamma(k+\tilde{N}+n+1 / 2)^{2}}{2^{2 k} \tilde{h}^{4 k}} \\
& \times \frac{h^{4 k} \cdot 2^{2 k} \cdot \Gamma(k+n / 2) \Gamma(k+n / 2+1 / 2)}{4^{4 k} \Gamma(k+(N+n) / 2) \Gamma(k+(N+n) / 2+1 / 4)} \\
& \times \frac{1}{\Gamma(k+(N+n) / 2+1 / 2) \Gamma(k+(N+n) / 2+3 / 4)},
\end{aligned}
$$

where $C(h, \tilde{h}, N, \tilde{N} ; n)>0$ is a constant not depending on the parameter $k$. If we put $2 \tilde{h}=h$ and $N=2 \tilde{N}+n / 2$, then

$$
\lim _{k \rightarrow \infty}\left\|T_{(\tilde{h}, \tilde{N} ; k)}^{\mathbb{C}}\right\| \quad \text { and } \quad \lim _{k \rightarrow \infty}\left\|T_{(\tilde{h}, \tilde{N} ; k)}^{\mathbb{C}}{ }^{-1}\right\|
$$

are both finite and non-zero. This proves the theorem since the norm of $T_{(\tilde{h}, \tilde{N})}^{\mathbb{C}}$ and its inverse are finite.

Proof of Theorem 6.9. Since the operator $T_{\left(h / 2, N_{0}\right)}^{\mathbb{C}} \circ T_{\left(h / 2, \tilde{N}_{0}\right)}^{\mathbb{C}}{ }^{-1}$ restricted to each space $E_{k}^{\mathbb{C}}$ $\left(N=2 N_{0}+n / 2\right.$ and $\left.\tilde{N}=2 \tilde{N}_{0}+n / 2\right)$ is

$$
\begin{aligned}
T_{\left(h / 2, N_{0}\right)}^{\mathbb{C}} \circ T_{\left(h / 2, \tilde{N}_{0}\right)}^{\mathbb{C}}-1 & \equiv \frac{b_{k}^{\mathbb{C}}\left(h / 2, \tilde{N}_{0}\right)}{b_{k}^{\mathbb{C}}\left(h / 2, N_{0}\right)} \mathrm{Id} \\
& =\frac{\Gamma\left(2 \tilde{N}_{0}+2 k+2 n\right)}{2^{k}(h / 2)^{2} \tilde{N}_{0}+2 k+2 n} \frac{2^{k}(h / 2)^{2 N_{0}+2 k+2 n}}{\Gamma\left(2 N_{0}+2 k+2 n\right)} \mathrm{Id} \\
& =h^{2\left(N_{0}-\tilde{N}_{0}\right)} \frac{\Gamma\left(2 \tilde{N}_{0}+2 k+2 n\right)}{\Gamma\left(2 N_{0}+2 k+2 n\right)} \mathrm{Id},
\end{aligned}
$$

we know by arguments similar to those in the proof of Theorem 3.5 that the composition $T_{\left(h / 2, N_{0}\right)}^{\mathbb{C}} \circ T_{\left(h / 2, \tilde{N}_{0}\right)}^{\mathbb{C}}{ }^{-1}$ is a pseudo-differential operator of order $\tilde{N}-N$. 
Now, we represent the reproducing kernel $\mathcal{K}_{(h, N)}^{\mathbb{C}}(A, B)$ of $\mathcal{H}^{2}\left(\mathbf{X}_{\mathbb{C}}, d m_{(h, N)}(A)\right)$ by an infinite sum and examine the asymptotic behavior of $A \mapsto \mathcal{K}_{(h, N)}^{\mathbb{C}}(A, A)$.

Since

$$
\frac{1}{a_{k}^{\mathbb{C}}(h, N)} A_{k}^{\mathbb{C}} \circ B_{(h, N ; k)}^{\mathbb{C}} \quad \text { and } \quad \frac{1}{c_{k}^{\mathbb{C}}(h, N)} C_{(h, N ; k)}^{\mathbb{C}}
$$

are the identity operators on the space $\mathcal{P}_{k}^{\mathbb{C}}\left(\mathbf{X}_{\mathbb{C}}\right)$, their kernel functions coincide and we conclude that

$$
\frac{1}{a_{k}^{\mathbb{C}}(h, N)} \int_{P^{n} \mathbb{C}}\left(\operatorname{tr}(P \cdot A) \cdot \operatorname{tr}\left(P \cdot B^{*}\right)\right)^{k} d \operatorname{vol}_{\mathbb{C}}(P)=\frac{1}{c_{k}^{\mathbb{C}}(h, N)}\left(\operatorname{tr}\left(A \cdot B^{*}\right)\right)^{k} .
$$

Then the power series

$$
\begin{aligned}
\sum_{k=0}^{\infty} \frac{\operatorname{dim} E_{k}^{\mathbb{C}} \lambda^{k}}{\Gamma(2 N+4 k+2 n)}= & \frac{1}{\Gamma(2 N+2 n)}+\sum_{k=1}^{\infty} n(n+2 k) \\
& \cdot\left(\frac{n(n+1) \cdots(n+k-1)}{k !}\right)^{2} \frac{\lambda^{k}}{\Gamma(2 N+4 k+2 n)}
\end{aligned}
$$

converges for any $\lambda \in \mathbb{C}$ and we can express the reproducing kernel $\mathcal{K}_{(h, N)}^{\mathbb{C}}(A, B)$ of the Hilbert space $\mathcal{H}^{2}\left(\mathbf{X}_{\mathbb{C}}, d m_{(h, N)}(A)\right)$ in the form of an infinite series by using the explicit formulas of the constants $\operatorname{dim} E_{k}^{\mathbb{C}}, a_{k}^{\mathbb{C}}(h, N)$ and $c_{k}^{\mathbb{C}}(h, N)$.

Proposition 7.4. We have

$$
\begin{aligned}
\mathcal{K}_{(h, N)}^{\mathbb{C}}(A, B) & =\sum_{k=0}^{\infty} \frac{1}{c_{k}^{\mathbb{C}}(h, N)}\left(\operatorname{tr}\left(A \cdot B^{*}\right)\right)^{k} \\
& =\frac{h^{2 N+2 n}}{\operatorname{Vol}(\Sigma(\mathbb{C}))} \cdot \sum_{k=0}^{\infty} \frac{\operatorname{dim} E_{k}^{\mathbb{C}} \cdot h^{4 k}}{\Gamma(2 N+4 k+2 n)}\left(\operatorname{tr}\left(A \cdot B^{*}\right)\right)^{k} \\
& =\sum_{k=0}^{\infty} \frac{1}{a_{k}^{\mathbb{C}}(h, N)} \int_{P^{n} \mathbb{C}}(\operatorname{tr}(P \cdot A))^{k}\left(\operatorname{tr}\left(P \cdot B^{*}\right)\right)^{k} d \operatorname{vol}_{\mathbb{C}}(P) .
\end{aligned}
$$

Note that $\mathcal{K}_{(h, N)}^{\mathbb{C}}(A, B)$ is defined for all matrices $A, B \in M(n+1, \mathbb{C})$, holomorphic with respect to the variable $A \in M(n+1, \mathbb{C})$ and anti-holomorphic with respect to the variable $B \in M(n+1, \mathbb{C})$. In particular,

$$
\mathcal{K}_{(h, N)}^{\mathbb{C}}(A, 0) \equiv \frac{1}{c_{k}^{\mathbb{C}}}=\frac{h^{2 N+2 n}}{\operatorname{Vol}(\Sigma(\mathbb{C})) \Gamma(2 N+2 n)} \equiv \mathcal{K}_{(h, N)}^{\mathbb{C}}(0, B) .
$$

THEOREM 7.5. Let $N>-n$, then

$$
\lim _{\|A\| \rightarrow \infty} \mathcal{K}_{(h, N)}^{\mathbb{C}}(A, A) e^{-h \sqrt{\|A\|}}\|A\|^{N}=2^{1-2 n} \cdot \frac{h^{2 n}}{\Gamma(n) \Gamma(n-1) \operatorname{Vol}(\Sigma(\mathbb{C}))} .
$$

Remark 7.6. By this property we know that a function on $\mathbf{X}_{\mathbb{C}}$ is $L_{2}$ with respect to the Liouville volume form if and only if it is $L_{2}$ with respect to the measure $\mathcal{K}_{(h, N)}^{\mathbb{C}}(A, A) d m_{(h, N)}(A)$ for any $N>-n$. Hence, we know that a Hankel operator on $\mathbf{X}_{\mathbb{C}}$ (and also $\mathbf{X}_{S}$ ) defined by a $L_{2}$-function with respect to the Liouville volume form $\Omega_{\mathbb{C}}$ is 
a Hilbert-Schmidt class operator, following a theory in [3] and [4]. These aspects will be discussed more precisely in [5] together with a study of invariant functions under Berezin transformation.

Proof. Put

$$
D(h, n, N)=\frac{h^{2 n+2 N} \cdot n}{\operatorname{Vol}(\Sigma(\mathbb{C})) \cdot \Gamma(n)^{2}},
$$

then

$$
\begin{aligned}
\mathcal{K}_{(h, N)}^{\mathbb{C}}(A, A)= & D(h, n, N) \sum \frac{(n+2 k) \Gamma(n+k)^{2}}{\Gamma(4 k+2 N+2 n)} \cdot \frac{\left(h^{2}\|A\|\right)^{2 k}}{(k !)^{2}} \\
= & 2 \cdot D(h, n, N) \sum \frac{\Gamma(k+n)^{2}}{\Gamma(4 k+2 n+2 N) \Gamma(k) \Gamma(k+1)} \cdot x^{4 k} \\
& +n \cdot D(h, n, N) \sum \frac{\Gamma(k+n)^{2}}{\Gamma(4 k+2 n+2 N) \Gamma(k+1)^{2}} \cdot x^{4 k},
\end{aligned}
$$

where $h \sqrt{\|A\|}=x$. Consider (7.7) modulo the constant multiple $2 \cdot D(h, n, N)$ :

$$
2 \cdot D(h, n, N) \sum \frac{\Gamma(k+n)^{2}}{\Gamma(4 k+2 n+2 N) \Gamma(k) \Gamma(k+1)} \cdot x^{4 k} .
$$

Let $k_{0} \in \mathbb{N}$ such that $2 N+4 k_{0}>0$. Then we decompose the term (7.7) by splitting it into two infinite sums

$$
\begin{aligned}
\sum & \frac{\Gamma(k+n)^{2}}{\Gamma(4 k+2 n+2 N) \Gamma(k) \Gamma(k+1)} \cdot x^{4 k} \\
= & \sum_{k<k_{0}} \frac{\Gamma(k+n)^{2}}{\Gamma(4 k+2 n+2 N) \Gamma(k) \Gamma(k+1)} \cdot x^{4 k} \\
& \quad+x^{4 k_{0}} \cdot \sum_{\ell \geq 0} \frac{\Gamma\left(\ell+k_{0}+n\right)^{2}}{\Gamma\left(4 \ell+4 k_{0}+2 n+2 N\right) \Gamma\left(\ell+k_{0}\right) \Gamma\left(\ell+k_{0}+1\right)} \cdot x^{4 \ell} .
\end{aligned}
$$

Let $A=4 k_{0}+2 N$ and $B=4^{4 k_{0}+2 n+2 N-1}$, then

$$
\lim _{\ell \rightarrow \infty} \frac{\Gamma\left(\ell+k_{0}+n\right)^{2} B}{\Gamma\left(4 \ell+4 k_{0}+2 n+2 N\right) \Gamma\left(\ell+k_{0}\right) \Gamma\left(\ell+k_{0}+1\right)} \frac{\Gamma(\ell+A+1) \Gamma(4 \ell+1)}{\Gamma(\ell+1)}=1 .
$$

As in the proof of Theorem 4.2 we consider the asymptotic behavior of the following infinite series

$$
\begin{aligned}
e^{-x} & x^{2 N+4 k_{0}} \sum_{\ell \geq 0} \frac{1}{B \cdot \Gamma(A)} \frac{\Gamma(\ell+1) \Gamma(A)}{\Gamma(\ell+A+1)} \frac{x^{4 \ell}}{(4 \ell) !} \\
& =e^{-x} x^{2 N+4 k_{0}} \frac{1}{B \cdot \Gamma(A)} \sum_{\ell \geq 0} \int_{0}^{1} t^{\ell}(1-t)^{A-1} d t \frac{x^{4 \ell}}{(4 \ell) !} \\
& =\frac{1}{B \cdot \Gamma(A)} e^{-x} x^{2 N+4 k_{0}} \int_{0}^{1}(1-t)^{A-1} \cdot \frac{\cosh \sqrt[4]{t} x+\cos \sqrt[4]{t} x}{2} d t .
\end{aligned}
$$


Now, it can be easily seen that we only need to consider the term

$$
e^{-x} x^{2 N+4 k_{0}} \int_{0}^{1}(1-t)^{A-1} \cdot \frac{e^{\sqrt[4]{t} x}}{4} d t .
$$

It follows by a coordinate change similar to that in the proof of Theorem 4.2 that

$$
\lim _{x \rightarrow \infty} e^{-x} x^{2 N+4 k_{0}} \int_{0}^{1}(1-t)^{A-1} \cdot e^{\sqrt[4]{t} x} d t=2^{8 k_{0}+4 N} \Gamma\left(4 k_{0}+2 N\right) .
$$

As for the term (7.8) we have

$$
e^{-x} x^{2 N} \sum \frac{\Gamma(k+n)^{2}}{\Gamma(4 k+2 n+2 N) \Gamma(k+1)^{2}} \cdot x^{4 k}=O\left(\frac{1}{x}\right) .
$$

Hence, (7.6) is proved.

Finally, in this section we state theorems corresponding to Theorems 5.1 and 5.2 on a quantization of the geodesic flow on the complex projective space.

Let $\left\{g_{t}^{\mathbb{C}}\right\}_{t \in \mathbb{R}}$ be the geodesic flow action (cf. [14]) on $\mathcal{H}^{2}\left(\mathbf{X}_{\mathbb{C}}, d m_{(h, N)}(A)\right.$ ), i.e. for a function $\varphi=\sum_{k=0}^{\infty} \varphi_{k} \in \mathcal{H}^{2}\left(\mathbf{X}_{\mathbb{C}}, d m_{(h, N)}(A)\right)$ with homogeneous $\varphi_{k}$ of degree $k$,

$$
g_{t}^{\mathbb{C}}(\varphi)(A)=\varphi\left(e^{\sqrt{-1} t} \cdot A\right)=\sum e^{\sqrt{-1} t} \varphi_{k}(A) .
$$

Let $\Delta_{P^{n}} \mathbb{C}$ be the Laplace operator on the complex projective space $P^{n} \mathbb{C}$. As a result corresponding to Theorem 5.1 we make the following remark.

THEOREM 7.7. We remark that

$$
T_{\left(h_{0}, N_{0}\right)}^{\mathbb{C}} \circ g_{t}^{\mathbb{C}}=e^{-(t \sqrt{-1} n) / 2} e^{(\sqrt{-1} t) / 2 \sqrt{\Delta_{P^{n} \mathbb{C}}+n^{2}}} \circ T_{\left(h_{0}, N_{0}\right)}^{\mathbb{C}},
$$

where

$$
T_{\left(h_{0}, N_{0}\right)}^{\mathbb{C}}: \mathcal{H}^{2}\left(\mathbf{X}_{\mathbb{C}}, d m_{(h, N)}(A)\right) \rightarrow L_{2}\left(P^{n} \mathbb{C}\right)
$$

and $h_{0}=h / 2$ and $N_{0}=1 / 2\{N-n / 2\}$ are given in Theorem 6.8 .

For each point $A \in \mathbf{X}_{\mathbb{C}}$, let $q_{A}^{\mathbb{C}}(P) \in L_{2}\left(P^{n} \mathbb{C}\right)$ be the function given by the Riesz' representation theorem:

$$
\begin{aligned}
T_{\left(h_{0}, N_{0}\right)}^{\mathbb{C}}{ }^{-1}(f)(A) & =\int_{\mathbf{X}_{\mathbb{C}}} T_{\left(h_{0}, N_{0}\right)}^{\mathbb{C}}{ }^{-1}(f)(B) \cdot \overline{\mathcal{K}_{(h, N)}^{\mathbb{C}}(B, A)} \cdot d m_{(h, N)}(B) \\
& =\int_{P^{n} \mathbb{C}} f(P) \cdot \overline{q_{A}^{\mathbb{C}}(P)} d \operatorname{vol}_{\mathbb{C}}(P),
\end{aligned}
$$

then

$$
q_{A}^{\mathbb{C}}(P)=\sum_{k=0}^{\infty} \frac{1}{b_{k}^{\mathbb{C}}\left(h_{0}, N_{0}\right)} \operatorname{tr}\left(A^{*} \cdot P\right)^{k},
$$

where $P=\left(p_{i} \bar{p}_{j}\right), p \in \mathbb{C}^{n+1}$ and $\|p\|=1$. Now, we have the following.

THEOREM 7.8. We have

$$
q_{e^{\sqrt{-1} t} \cdot A}^{\mathbb{C}}=e^{(t \sqrt{-1} n) / 2} e^{-\sqrt{-1} t / 2 \sqrt{\Delta_{P n} \mathbb{C}+n^{2}}}\left(q_{A}^{\mathbb{C}}\right) .
$$




\section{Complexified Hopf fibration and quantization operators}

Recall the $\mathbb{C}^{*}$-principal bundle (we call it the complexified Hopf bundle):

$$
\alpha: \mathbf{X}_{S}^{0} \rightarrow \mathbf{X}_{\mathbb{C}}
$$

Consider the $(4 n+2)$-form on $\mathbf{X}_{S}^{0}$ given by $e^{-h \sqrt{\|u\|^{2}+\|v\|^{2}}} \cdot\left(\|u\|^{2}+\|v\|^{2}\right)^{N / 2} \Omega_{S}$. In this section first we determine the fiber integral

$$
\alpha_{*}\left(e^{-h \sqrt{\|u\|^{2}+\|v\|^{2}}} \cdot\left(\|u\|^{2}+\|v\|^{2}\right)^{N / 2} \Omega_{S}\right),
$$

and then we discuss the closeness of the image of the operator

$$
\alpha^{*}: \mathcal{H}^{2}\left(\mathbf{X}_{\mathbb{C}}, d m_{\left(h_{\mathbb{C}}, N_{\mathbb{C}}\right.}(A)\right) \rightarrow \mathcal{H}^{2}\left(\mathbf{X}_{S}, d m_{\left(h_{S}, N_{S}\right)}(u, v)\right) .
$$

We work on

$$
\begin{aligned}
& \beta: \gamma\left(\mathbf{X}_{S}^{0}\right)=\left\{(z, w) \in \mathbb{C}^{n+1} \times \mathbb{C}^{n+1} \mid z \neq 0, w \neq 0, \sum z_{i} w_{i}=0\right\} \rightarrow \mathbf{X}_{\mathbb{C}}, \\
& \beta:(z, w) \mapsto\left(z_{i} w_{j}\right) .
\end{aligned}
$$

PROPOSITION 8.1. We have

$$
\begin{aligned}
& \beta_{*}\left(e^{-h / \sqrt{2} \sqrt{\|z\|^{2}+\|w\|^{2}}} \cdot\left(\frac{\|z\|^{2}+\|w\|^{2}}{2}\right)^{N / 2} \Omega_{S}\right)(A) \\
& \quad=\frac{\pi \cdot 2^{n+1}}{2^{N / 2}}\|A\|^{n} I(\|A\|) \Omega_{\mathbb{C}}(A),
\end{aligned}
$$

where

$$
I(\|A\|)=\int_{0}^{\infty} e^{-(h / \sqrt{2}) \sqrt{r^{2}+(\|A\| / r)^{2}}}\left(r^{2}+(\|A\| / r)^{2}\right)^{(N+1) / 2-n} \frac{d r}{r} .
$$

The formula (8.1) above was proved in [15]. For the sake of the completeness, we give a simple proof here. We use the same notation for the Liouville volume form $\Omega_{S}$ (respectively $\Omega_{\mathbb{C}}$ ) and its pull-back through the map $\left(\tau_{S} \circ \gamma\right)^{-1}$ (respectively $\left.\left(\tau_{\mathbb{C}}\right)^{-1}\right)$. Also note that $2\left(\|u\|^{2}+\|v\|^{2}\right)=\|z\|^{2}+\|w\|^{2}$ for $\gamma(u, v)=(z, w)$.

Proof. Since the unitary group $U(n+1)$ acts on

$$
\mathbf{X}_{\mathbb{C}} \cap\left\{A \in M(n+1, \mathbb{C}) \mid \operatorname{tr}\left(A \cdot A^{*}\right)=1\right\}
$$

transitively (adjoint action : $A \mapsto U A U^{*}, U \in U(n+1)$ ) and the Liouville volume form $\Omega_{\mathbb{C}}$ is invariant under this action together with the equivariance between the Liouville volume form on $\mathbf{X}_{S}$ under the action of the unitary group $U(n+1)$ on $\mathbb{C}^{n+1} \times \mathbb{C}^{n+1}$ by $(z, w) \mapsto(U(z), \bar{U}(w))$, it is enough to determine the fiber integration on the points

$$
A_{t}=\left(\begin{array}{cccc}
0 & t & 0 & 0 \\
0 & 0 & \cdots & 0 \\
\vdots & \vdots & \ddots & \vdots \\
0 & \cdots & \cdots & 0
\end{array}\right) \in \mathbf{X}_{\mathbb{C}}, \quad t>0
$$


Note that $\mathbf{X}_{S}^{0}$ is invariant under the action $(z, w) \mapsto(U(z), \bar{U}(w))(\bar{U}$ is the unitary matrix whose entries are the complex conjugates of those of $U$ ).

Then we take a local coordinate system on an open subset of $\mathbf{X}_{\mathbb{C}}$ on which, incidentally, we have a local trivialization of the map $\beta$ :

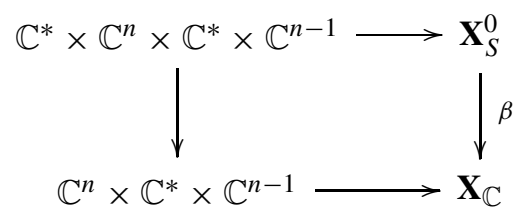

such that

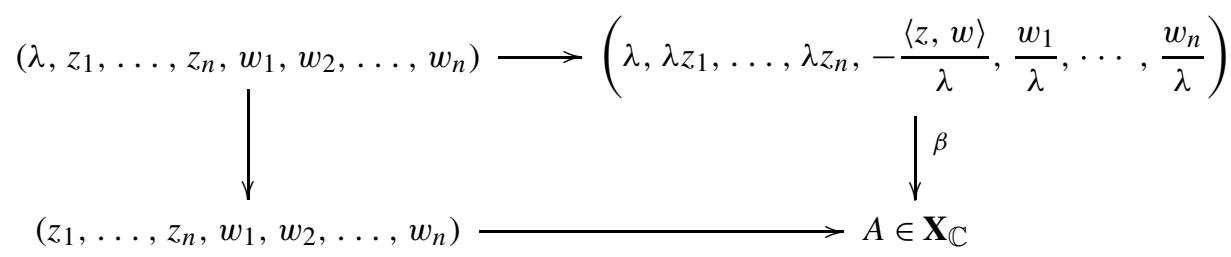

where $w_{1} \neq 0,\langle z, w\rangle=\sum_{i=1}^{n} z_{i} w_{i}$ and

$$
A=\left(\begin{array}{ccccc}
-\langle z, w\rangle & w_{1} & w_{2} & \cdots & w_{n} \\
-z_{1}\langle z, w\rangle & z_{1} w_{1} & z_{1} w_{2} & \cdots & z_{1} w_{n} \\
\vdots & \vdots & \vdots & \ddots & \vdots \\
-z_{n}\langle z, w\rangle & z_{n} w_{1} & z_{n} w_{2} & \cdots & z_{n} w_{n}
\end{array}\right)
$$

In terms of these coordinates $\omega_{S}$ can be written as

$$
\omega_{S}=\sqrt{-1} \bar{\partial} \partial\left(\sqrt{|\lambda|^{2}\left(1+\|z\|^{2}\right)+\frac{|\langle z, w\rangle|^{2}+\|w\|^{2}}{|\lambda|^{2}}}\right) .
$$

When we expand

$$
\begin{aligned}
4 \cdot\left(|\lambda|^{2}+\frac{t^{2}}{|\lambda|^{2}}\right)^{3 / 2} \cdot \omega_{S}= & a_{00} d \bar{\lambda} \wedge d \lambda+\sum a_{0, k} d \bar{z}_{k} \wedge d \lambda+\sum b_{0, k} d \bar{w}_{k} \wedge d \lambda \\
& +\sum \bar{a}_{0 j} d \bar{\lambda} \wedge d z_{j}+\sum a_{j k} d \bar{z}_{k} \wedge d z_{j} \\
& +\sum b_{j k} d \bar{w}_{k} \wedge d z_{j}+\sum \bar{b}_{0 j} d \bar{\lambda} \wedge d w_{j} \\
& +\sum \bar{b}_{k j} d \bar{z}_{k} \wedge d w_{j}+\sum c_{j k} d \bar{w}_{k} \wedge d w_{j},
\end{aligned}
$$


then by making use of the expression (8.2) we can calculate the values $a_{k, j}, b_{k, j}$ and $c_{k, j}$ at the point $\left(\lambda, z, w_{1}, w_{2}, \ldots, w_{n}\right)=(\lambda, 0, t, 0, \ldots, 0)$ explicitly as follows:

$$
\begin{aligned}
& a_{00}=|\lambda|^{2}+\frac{6 t^{2}}{|\lambda|^{2}}+\frac{t^{4}}{|\lambda|^{6}}, \quad a_{0 k}=0 \quad(k \geq 1), \\
& b_{01}=-\frac{t}{\lambda|\lambda|^{2}}\left(3|\lambda|^{2}+\frac{t^{2}}{|\lambda|^{2}}\right), \quad b_{0 k}=0 \quad(k \geq 2), \\
& b_{k j}=0 \quad(\text { for all } k \geq 1 \text { and all } j \geq 1), \\
& a_{11}=2\left(|\lambda|^{2}+\frac{t^{2}}{|\lambda|^{2}}\right)^{2}, \quad a_{k k}=2\left(|\lambda|^{2}+\frac{t^{2}}{|\lambda|^{2}}\right)|\lambda|^{2} \quad(k \geq 2), \quad a_{j k}=0 \quad(k \neq j), \\
& c_{11}=2+\frac{t^{2}}{|\lambda|^{4}}, \quad c_{k k}=2\left(|\lambda|^{2}+\frac{t^{2}}{|\lambda|^{2}}\right) \cdot \frac{1}{|\lambda|^{2}} \quad(k \geq 2), \quad c_{j k}=0 \quad(k \neq j) .
\end{aligned}
$$

Put

$$
D(\lambda, t)=\left(\begin{array}{ccccccc}
a_{00} & a_{01} & \cdots & a_{0 n} & b_{01} & \cdots & b_{0 n} \\
\bar{a}_{01} & a_{11} & \cdots & a_{1 n} & b_{11} & \cdots & b_{1 n} \\
\vdots & \vdots & \ddots & \vdots & \vdots & \ddots & \vdots \\
\bar{a}_{0 n} & \bar{a}_{1 n} & \cdots & a_{n n} & b_{n 1} & \cdots & b_{n n} \\
\bar{b}_{01} & \bar{b}_{11} & \cdots & \bar{b}_{n 1} & c_{11} & \cdots & c_{1 n} \\
\vdots & \vdots & \ddots & \vdots & \vdots & \ddots & \vdots \\
\bar{b}_{0 n} & \bar{b}_{1 n} & \cdots & \bar{b}_{n n} & \bar{c}_{1 n} & \cdots & c_{n n}
\end{array}\right),
$$

then we can express the Liouville volume form $\Omega_{S}$ by the coordinates $(\lambda, z, w)$ :

$$
\begin{aligned}
\Omega_{S}= & \frac{(-1)^{n(2 n+1)}}{(2 n+1) !} \omega_{S}^{2 n+1} \\
= & \frac{(-1)^{n(2 n+1)}}{(2 n+1) !} \cdot(-1)^{n} \sqrt{-1} \cdot\left(\frac{1}{4\left(|\lambda|^{2}+t^{2} /|\lambda|^{2}\right)^{3 / 2}}\right)^{2 n+1} \cdot(2 n+1) ! \cdot \operatorname{det} D(\lambda, t) \\
& d \bar{\lambda} \wedge d \lambda \wedge d \bar{z}_{1} \wedge d z_{1} \wedge \cdots \wedge d \bar{z}_{n} \wedge d z_{n} \wedge d \bar{w}_{1} \wedge d w_{1} \wedge \cdots \wedge d \bar{w}_{n} \wedge d w_{n} \\
= & \sqrt{-1} \cdot\left(\frac{1}{4\left(|\lambda|^{2}+t^{2} /|\lambda|^{2}\right)^{3 / 2}}\right)^{2 n+1} \\
& \times\left(\left(|\lambda|^{2}+\frac{6 t^{2}}{|\lambda|^{2}}+\frac{t^{4}}{|\lambda|^{6}}\right) \cdot\left(2+\frac{t^{2}}{|\lambda|^{4}}\right)-\frac{t^{2}}{|\lambda|^{6}}\left(3|\lambda|^{2}+\frac{t^{2}}{|\lambda|^{2}}\right)^{2}\right) \\
& \times 2\left(|\lambda|^{2}+\frac{t^{2}}{|\lambda|^{2}}\right)^{2} \cdot\left(2\left(|\lambda|^{2}+\frac{t^{2}}{|\lambda|^{2}}\right)|\lambda|^{2}\right)^{n-1} \cdot\left(2\left(|\lambda|^{2}+\frac{t^{2}}{|\lambda|^{2}}\right) \frac{1}{|\lambda|^{2}}\right)^{n-1} \\
& d \bar{\lambda}^{2} \wedge d \lambda \wedge d \bar{z}_{1} \wedge d z_{1} \wedge \cdots \wedge d \bar{z}_{n} \wedge d z_{n} \wedge d \bar{w}_{1} \wedge d w_{1} \wedge \cdots \wedge d \bar{w}_{n} \wedge d w_{n} \\
= & \frac{\sqrt{-1}}{2^{2 n+2}|\lambda|^{2}} \cdot\left(\frac{1}{|\lambda|^{2}+t^{2} /|\lambda|^{2}}\right)^{n-1 / 2} d \bar{\lambda} \wedge d \lambda \wedge d \bar{z}_{1} \wedge d z_{1} \wedge \cdots \wedge d \bar{z}_{n} \wedge d z_{n} \\
& \wedge d \bar{w}_{1} \wedge d w_{1} \wedge \cdots \wedge d \bar{w}_{n} \wedge d w_{n} .
\end{aligned}
$$


Hence, at the point

$$
A_{t}=\left(\begin{array}{cccc}
0 & t & 0 & 0 \\
0 & 0 & \cdots & 0 \\
\vdots & \vdots & \ddots & \vdots \\
0 & \cdots & \cdots & 0
\end{array}\right) \in \mathbf{X}_{\mathbb{C}}, \quad t>0
$$

the push-forward of the $(4 n+2)$-form $e^{-h \sqrt{\|u\|^{2}+\|v\|^{2}}}\left(\|u\|^{2}+\|v\|^{2}\right)^{N / 2} \Omega_{S}$ by the map $\beta$ is

$$
\begin{aligned}
& \beta_{*}\left(e^{-h \sqrt{\|u\|^{2}+\|v\|^{2}}}\left(\|u\|^{2}+\|v\|^{2}\right)^{N / 2} \Omega_{S}\right)\left(A_{t}\right) \\
& =\frac{\pi}{2^{2 n}} \cdot \int_{0}^{\infty} \frac{1}{r}\left(\frac{1}{r^{2}+(t / r)^{2}}\right)^{n-1 / 2} e^{-h / \sqrt{2} \sqrt{r^{2}+(t / r)^{2}}}\left(\frac{r^{2}+(t / r)^{2}}{2}\right)^{N / 2} \\
& \quad d r d \bar{z}_{1} \wedge d z_{1} \wedge \cdots \wedge d \bar{z}_{n} \wedge d z_{n} \wedge d \bar{w}_{1} \wedge d w_{1} \wedge \cdots \wedge d \bar{w}_{n} \wedge d w_{n} .
\end{aligned}
$$

Next, the Liouville volume form

$$
\Omega_{\mathbb{C}}=\frac{(-1)^{n(2 n-1)}}{(2 n) !}(\sqrt{-2} \bar{\partial} \partial \sqrt{\|A\|})^{2 n}
$$

is expressed around the point $\left(0, \ldots, 0, w_{1}, 0, \ldots, 0\right)$ (we put $\left.w_{1}=t>0\right)$ in the local coordinates $(z, w)$ as

$$
\Omega_{\mathbb{C}}\left(A_{t}\right)=\frac{1}{2^{3 n+1} \cdot t^{n}} d \bar{z}_{1} \wedge d z_{1} \wedge \cdots \wedge d \bar{z}_{n} \wedge d z_{n} \wedge d \bar{w}_{1} \wedge d w_{1} \wedge \cdots \wedge d \bar{w}_{n} \wedge d w_{n} .
$$

By comparing (8.3) and (8.4) we have (8.1).

Let $f \in \mathcal{P}_{k}\left(\mathbf{X}_{\mathbb{C}}\right)$ be a polynomial of degree $k$ in the variable $A \in M(n+1, \mathbb{C})$, then $\alpha^{*}(f)(u, v)$ is a $2 k$-degree polynomial on $\mathbb{C}^{n+1} \times \mathbb{C}^{n+1}$. Since $\alpha^{*}(f)(u, v)$ vanishes on $\mathbf{X}_{S} \backslash \mathbf{X}_{S}^{0}$, the $L_{2}$-norm of

$$
\alpha^{*}(f)(u, v)=\gamma^{*}\left(\beta^{*}(f)\right)(u, v)
$$

with respect to the measure $e^{-h \sqrt{\|u\|^{2}+\|v\|^{2}}}\left(\|u\|^{2}+\|v\|^{2}\right)^{N / 2} \Omega_{S}$ is

$$
\begin{aligned}
\int_{\mathbf{X}_{S}}\left|\alpha^{*}(f)\right|^{2} e^{-h \sqrt{\|u\|^{2}+\|v\|^{2}}}\left(\|u\|^{2}+\|v\|^{2}\right)^{N / 2} \Omega_{S} \\
=\int_{\mathbf{X}_{S}^{0}}\left|\alpha^{*}(f)\right|^{2} e^{-h \sqrt{\|u\|^{2}+\|v\|^{2}}}\left(\|u\|^{2}+\|v\|^{2}\right)^{N / 2} \Omega_{S} \\
=F(n, N) \int_{\mathbf{X}_{\mathbb{C}}} \int_{0}^{\infty}|f(A)|^{2}\|A\|^{n} \cdot e^{-\left(h_{S} / \sqrt{2}\right) \sqrt{r^{2}+(\|A\| / r)^{2}}} \\
\quad \times\left(r^{2}+(\|A\| / r)^{2}\right)^{(N+1) / 2-n} \frac{d r}{r} \wedge \Omega_{\mathbb{C}}(A) \\
=F(n, N) \int_{\Sigma(\mathbb{C})}|f(A /\|A\|)|^{2} d \mathrm{v}_{\Sigma(\mathbb{C})}(\sigma) \\
\quad \times \int_{0}^{\infty} \int_{0}^{\infty} t^{2 k} \cdot t^{n} \cdot e^{-(h / \sqrt{2})} \sqrt{r^{2}+(t / r)^{2}} \cdot\left(r^{2}+(t / r)^{2}\right)^{(N+1) 2-n} \cdot t^{n-1} \frac{d r \wedge d t}{r}
\end{aligned}
$$




$$
\begin{aligned}
= & F(n, N) \cdot \int_{\Sigma(\mathbb{C})}|f(A /\|A\|)|^{2} d \mathrm{v}_{\Sigma(\mathbb{C})}(\sigma) \\
& \times \frac{2^{n+(N-1) / 2}}{h^{2 n+N+1}} \cdot \frac{2^{2 k}}{h^{4 k}} \cdot \frac{\Gamma(4 k+2 n+N+1) \cdot \Gamma(k+n)^{2}}{\Gamma(2 k+2 n)} \\
= & G(n, N) \cdot \int_{\Sigma(\mathbb{C})}|f(A /\|A\|)|^{2} d \mathrm{v}_{\Sigma(\mathbb{C})}(\sigma) \cdot \frac{2^{2 k}}{h^{4 k}} \cdot \frac{\Gamma(4 k+2 n+N+1) \cdot \Gamma(k+n)^{2}}{\Gamma(2 k+2 n)},
\end{aligned}
$$

where we put

$$
F(n, N)=\frac{\pi \cdot 2^{n}}{2^{N / 2}} \quad \text { and } \quad G(n, N)=F(n, N) \cdot \frac{2^{n+(N-1) / 2}}{h^{2 n+N+1}} .
$$

Next, we express the $L_{2}$-norm of a degree $k$ polynomial $f \in \mathcal{P}_{k}(M(n+1, \mathbb{C}))$ with respect to the measure $e^{-h \sqrt{\|A\|}}\|A\|^{N} \Omega_{\mathbb{C}}$ :

$$
\int_{\mathbf{X}_{\mathbb{C}}}|f(A)|^{2} e^{-h \sqrt{\|A\|}}\|A\|^{N} \Omega_{\mathbb{C}}=\frac{2}{h^{2 n+2 N}} \cdot \int_{\Sigma(\mathbb{C})}|f(\sigma)|^{2} d v_{\Sigma(\mathbb{C})}(\sigma) \cdot \frac{\Gamma(4 k+2 n+2 N)}{h^{4 k}} .
$$

We have the following.

THEOREM 8.2. Let $h_{S}=h_{\mathbb{C}}>0$ and $N_{S}=2 N_{\mathbb{C}}-\frac{1}{2}$ with $N_{\mathbb{C}}>-n, N_{S}>-2 n-1$, then the map

$$
\alpha^{*}: \mathcal{H}^{2}\left(\mathbf{X}_{\mathbb{C}}, d m_{\left(h_{\mathbb{C}}, N_{\mathbb{C}}\right)}(A)\right) \rightarrow \mathcal{H}^{2}\left(\mathbf{X}_{S}, d m_{\left(h_{S}, N_{S}\right)}(u, v)\right)
$$

is continuous, and the image $\alpha^{*}\left(\mathcal{H}^{2}\left(\mathbf{X}_{\mathbb{C}}, d m_{\left(h_{\mathbb{C}}, N_{\mathbb{C}}\right)}\right)\right)$ is a closed subspace of the Hilbert space $\mathcal{H}^{2}\left(\mathbf{X}_{S}, d m_{\left(h_{S}, N_{S}\right)}(u, v)\right)$.

Proof. The square root of the ratio of (8.5) divided by (8.6) does not depend on the polynomials $f \in \mathcal{P}_{k}\left(\mathbf{X}_{\mathbb{C}}\right)$. It is the norm $\left\|\alpha_{\mid \mathcal{P}_{k}}\left(\mathbf{X}_{\mathbb{C}}\right)^{*}\right\|$ of the operator $\alpha^{*}$ restricted to each subspace $\mathcal{P}_{k}\left(\mathbf{X}_{\mathbb{C}}\right)$ and mapping to the subspace

$$
\mathcal{P}_{2 k}\left(\mathbf{X}_{S}\right) \subset \mathcal{H}^{2}\left(\mathbf{X}_{S}, d m_{\left(h_{S}, N_{S}\right)}(u, v)\right) .
$$

Moreover, we know that $\lim _{k \rightarrow \infty}\left\|\alpha_{\mid \mathcal{P}_{k}\left(\mathbf{x}_{\mathbb{C}}\right)}{ }^{*}\right\|$ is finite and non-zero by the same reason as in the proof of (3.1) and (3.2) and for $h_{S}=h_{\mathbb{C}}$ and $N_{S}=2 N_{\mathbb{C}}-2^{-1}$.

Again let $f \in \mathcal{P}_{k}(M(n+1, \mathbb{C}))$, then we have the following.

Proposition 8.3. The pull-back of the function $f$ by the map $\alpha, \alpha^{*}(f)(u, v)$, is a $2 k$-degree polynomial on $\mathbb{C}^{n+1} \times \mathbb{C}^{n+1}$, and the function

$$
\begin{aligned}
B_{(h, N ; 2 k)}^{S}\left(\alpha^{*}(f)\right)\left(x^{\prime}, x^{\prime \prime}\right)= & \int_{\mathbf{X}_{S}^{0}} \alpha^{*}(f)(u, v)\left(\left\langle x^{\prime}, \bar{u}\right\rangle+\left\langle x^{\prime \prime}, \bar{v}\right\rangle\right)^{2 k} \\
& \times e^{-h \sqrt{\|u\|^{2}+\|v\|^{2}}}\left(\|u\|^{2}+\|v\|^{2}\right)^{N / 2} \Omega_{S}(u, v)
\end{aligned}
$$

is a $U(1)$-invariant harmonic function. Its descent to $P^{n} \mathbb{C}$ (we write $\overline{\left.B_{(h, N ; 2 k)}^{S}(f)\right)}$ is an eigenfunction in $E_{k}^{\mathbb{C}}$ of the Laplacian on $P^{n} \mathbb{C}$. (It does not coincide with $B_{\left(h^{\prime}, N^{\prime} ; k\right)}^{\mathbb{C}}(f)$.) 
Conversely, let $g(x) \in \mathcal{S}_{2 k}\left(\mathbb{C}^{n+1}\right)$ be a harmonic polynomial of the variables $p_{i} \bar{p}_{j}$, i.e. it can be descended to the complex projective space, then we have the following.

Proposition 8.4. We have

$$
A_{2 k}^{S}(g)(u, v)=\int_{S^{2 n+1}} g(x)\left(\left\langle x^{\prime}, u\right\rangle+\left\langle x^{\prime \prime}, v\right\rangle\right)^{2 k} d \operatorname{vol}_{S}
$$

can be descended to $\mathbf{X}_{\mathbb{C}}$, that is, $A_{2 k}^{S}(g)$ is invariant under the action (6.2).

THEOREM 8.5. Let $N_{S}=2 N_{\mathbb{C}}-1 / 2, N_{\mathbb{C}}>-n$, then the operator

$$
T_{\left(h / 2, N_{\mathbb{C}}-(n+1) / 4\right)}^{S} \circ \alpha^{*}: \mathcal{H}^{2}\left(\mathbf{X}_{\mathbb{C}}, d m_{\left(h, N_{\mathbb{C}}\right)}(A)\right) \longrightarrow L_{2}\left(P^{n} \mathbb{C}\right)
$$

is an isomorphism and the operator

$$
T_{\left(h / 2, N_{\mathbb{C}}-(n+1) / 4\right)}^{S} \circ \alpha^{*} \circ T_{\left(h / 2, N_{\mathbb{C}} / 2-n / 4\right)}^{\mathbb{C}}{ }^{-1}
$$

is a zeroth-order elliptic pseudo-differential operator in $L_{1,0}^{0}\left(P^{n} \mathbb{C}\right)$.

Here $T_{\left(h / 2, N_{\mathbb{C}}-(n+1) / 4\right)}^{S} \circ \alpha^{*}(f)$ should be understood as a function descended to the complex projective space according to Proposition 8.3.

Remark 8.6. Although the determination of the values $N_{S}$ and $N_{\mathbb{C}}$ (also $h_{S}$ and $h_{\mathbb{C}}$ ) by the pairing of polarizations (cf. [23] and [15]) is preformed independently in each case, they satisfy the relation above.

Remark 8.7. In [13] we proved a similar property in the particular case of the parameter $\left(h_{S}, N_{S}\right)$ for the quaternion projective space.

Finally, we provide a relation between the reproducing kernels $\mathcal{K}_{\left(h, N_{S}\right)}^{S}(a, b ; u, v)$ and $\mathcal{K}_{\left(h, N_{\mathbb{C}}\right)}^{\mathbb{C}}(B, A)$, where $N_{S}=2 N_{\mathbb{C}}-1 / 2$. Let $f \in \mathcal{H}^{2}\left(\mathbf{X}_{\mathbb{C}}, d m_{\left(h, N_{\mathbb{C}}\right)}(A)\right)$, then

$$
\begin{aligned}
\alpha^{*}(f)(a, b) & =\int_{\mathbf{X}_{S}} \alpha^{*}(f)(u, v) \overline{\mathcal{K}_{\left(h, N_{S}\right)}^{S}(a, b ; u, v)} e^{-h \sqrt{\|u\|^{2}+\mid v \|^{2}}}\left(\|u\|^{2}+\|v\|^{2}\right)^{N_{S} / 2} \Omega_{S} \\
& =\int_{\mathbf{X}_{\mathbb{C}}} f(A) \overline{\mathcal{K}_{\left(h, N_{\mathbb{C}}\right)}^{\mathbb{C}}(\alpha(a, b), A)} e^{-h \sqrt{\|A\|}}\|A\|^{N_{\mathbb{C}} / 2} \Omega_{\mathbb{C}}=f(\alpha(a, b)) .
\end{aligned}
$$

Put

$$
\begin{aligned}
& \alpha_{*}\left(\mathcal{K}_{\left(h, N_{S}\right)}^{S}(a, b ; \bullet, \bullet) \cdot e^{-h \sqrt{\|\bullet\|^{2}+\|\bullet\|^{2}}}\left(\|\bullet\|^{2}+\|\bullet\|^{2}\right)^{N_{S} / 2} \Omega_{S}\right)(A) \\
& \quad=L(a, b ; A) \cdot e^{h \sqrt{\|A\|}}(\|A\|)^{-N_{\mathbb{C}} / 2} \Omega_{\mathbb{C}},
\end{aligned}
$$

then $L(a, b ; A)-\mathcal{K}_{\left(h, N_{\mathbb{C}}\right)}^{\mathbb{C}}(\alpha(a, b), A)$ is orthogonal to $\mathcal{H}^{2}\left(\mathbf{X}_{\mathbb{C}}, d m_{\left(h, N_{\mathbb{C}}\right)}(A)\right)$, and not zero. We have

$$
\int_{\mathbf{X}_{\mathbb{C}}} L(a, b ; A) \cdot \overline{\mathcal{K}_{\left(h, N_{\mathbb{C}}\right)}^{\mathbb{C}}(B, A)} \cdot e^{-h \sqrt{\|A\|}}\|A\|^{N_{\mathbb{C}} / 2} \Omega_{\mathbb{C}}=\mathcal{K}_{\left(h, N_{\mathbb{C}}\right)}^{\mathbb{C}}(\alpha(a, b), B) .
$$


Acknowledgements. WB was partially supported by a JSPS postdoctoral fellowship (No. PE05570) for North American and European researchers. KF was partially supported by the Grant-in-aid Scientific Research (C) No. 17540202, Japan Society for Promotion of Science.

\section{REFERENCES}

[1] G. E. Andrews, R. Askey and R. Roy. Special Functions (Encyclopedia of Mathematics and its Applications, 71). Cambridge University Press, Cambridge, 1999.

[2] V. Bargmann. Irreducible unitary representations of the Lorentz group. Ann. of Math. (2) 48 (1947), 568640.

[3] W. Bauer. Hilbert-Schmidt Hankel operators on the Segal-Bargmann space. Proc. Amer. Math. Soc. 132(10) (2004), 2989-2996.

[4] W. Bauer and K. Furutani. Hilbert-Schmidt Hankel Operators and Berezin iteration. Tokyo J. Math., to appear.

[5] W. Bauer and K. Furutani. Quantization operator on quaternion projective space and Cayley projective space. In preparation.

[6] M. Berger, P. Gauduchon and E. Mazet. Le Spectre dúne Variété Riemannienne (Lecture Notes in Mathematics, 194). Springer, Berlin, 1971.

[7] A. L. Besse. Manifolds all of whose Geodesics are Closed. Springer, Berlin, 1978.

[8] L. Boutet de Monvel and V. Guillemin. The spectral theory of Toeplitz operators (Annales of Mathematics Studies, 99). Princeton University Press, Princeton, NJ, 1981.

[9] M. de Gosson. On the Leray-Maslov quantization of Lagrangian manifold. J. Geom. Phys. 13 (1994), 155168.

[10] J. J. Duistermaat and V. Guillemin. The spectrum of positive elliptic operators and periodic bicharacteristics. Invent. Math. 29 (1975), 39-79.

[11] K. Furutani. Quantization of the geodesic flow on quaternion projective spaces. Annals Global Anal. Geom. 22(1) (2002), 1-27.

[12] K. Furutani. A Kähler structure on the punctured cotangent bundle of the Cayley projective plane. Math. Phys. Stud. 24 (2003), 163-182.

[13] K. Furutani. Pseudo-differential operator and reproducing kernels arising in geometric quantization. Operat. Theory Adv. Appl. 164 (2006), 133-151.

[14] K. Furutani and R. Tanaka. A Kähler structure on the punctured cotangent bundle of complex and quaternion projective spaces and its application to geometric quantization I. J. Math. Kyoto Univ. 34 (1994), 719-737.

[15] K. Furutani and S. Yoshizawa. A Kähler structure on the punctured cotangent bundle of complex and quaternion projective spaces and its application to geometric quantization II. Japan. J. Math. 21 (1995), $355-392$.

[16] V. Guillemin and S. Sternberg. Geometric Asymptotics (Mathematical Surveys and Monographs, 14). American Mathematical Society, 1977.

[17] L. Hörmander. Fourier integral operators I. Acta Math. 127 (1971), 79-183.

[18] L. Hörmander. The Analysis of Linear Partial Differential Operators III. Springer, Berlin, 1985.

[19] K. Ii. On a Bargmann-type transform and a Hilbert space of holomorphic functions, Tôhoku Math. J. 38 (1986), 57-69.

[20] K. Ii and T. Morikawa. Kähler structures on the tangent bundle of Riemannian manifolds of constant positive curvature. Bull. Yamagata Univ. Natur. Sci. 14 (1999), 141-154.

[21] W. Lichtenstein. A system of quadrics describing the orbit of the highest weight vector. Proc. Amer. Math. Soc. 84 (1982), 605-608.

[22] J. H. Rawnsley. Coherent states and Kähler manifolds. Quart. J. Math. Oxford Ser. 28 (1977), 403-415.

[23] J. H. Rawnsley. A non-unitary pairing of polarization for the Kepler problem. Trans. Amer. Math. Soc. 250 (1979), 167-180.

[24] J. M. Souriau. Sur la variété de Kepler. Symposia Math. 14 (1974), 343-360.

[25] R. Szőke. Complex structures on the tangent bundle of Riemannian manifolds, Math. Ann. 291 (1991), 409-428.

[26] R. Szőke. Adapted complex structures and geometric quantization. Nagoya J. Math. 154 (1999), 171-183.

[27] R. Szőke. Involutive structures on tangent bundles of symmetric spaces. Math. Ann. 319 (2001), 319-348. 
[28] M. Taylor. Pseudo-differential Operators (Princeton Mathematical Series, 34). Princeton University Press, Princeton, NJ, 1981.

[29] A. Weinstein, Fourier Integral Operators, Quantization and the Spectra of Riemannian Manifolds. Colloque International de Géométrie Symplectique et Physique Mathématique (Colloq. Internat. CNRS, No. 237, Aixen-Provence, 1974), pp. 289-298.

[30] N. M. J. Woodhouse. Geometric Quantization, 2nd edn (Oxford Mathematical Monographs). Clarendon Press, Oxford, 1997.

\author{
Wolfram Bauer \\ Fachbereich Mathematik und Informatik \\ Johannes-Gutenberg Universität \\ Staudinger Weg 9 \\ 55128 Mainz, Germany \\ (E-mail: BauerWolfram@web.de)
}

\title{
Kenro Furutani
}

Department of Mathematics

Faculty of Science and Technology

Science University of Tokyo

2641 Yamazaki, Noda, Chiba (278-8510), Japan

(E-mail: furutani_kenro@ma.noda.tus.ac.jp) 